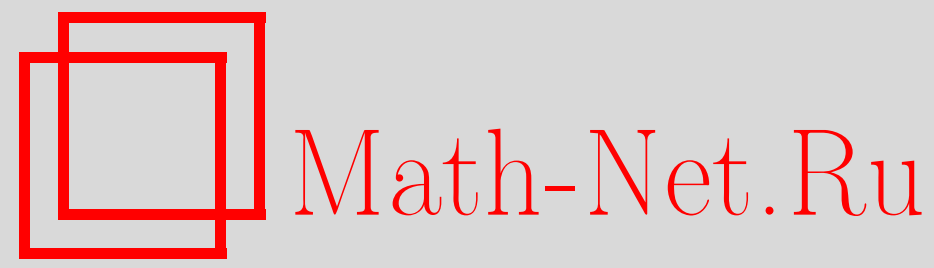

О. В. Камловский, Количество различных мультиграмм в линейных рекуррентных последовательностях над кольцами Галуа, Mатем. вопр. криптогр., 2013, том 4, выпуск 3, 49-82

DOI: https://doi.org/10.4213/mvk92

Использование Общероссийского математического портала Math-Net.Ru подразумевает, что вы прочитали и согласны с пользовательским соглашением

http: //www. mathnet.ru/rus/agreement

Параметры загрузки:

IP: 3.91 .87 .62

26 апреля 2023 г., 16:05:19 


\section{Количество различных мультиграмм в линейных рекуррентных последовательностях над кольцами Галуа

\author{
О. В. Камловский
}

ООО «Центр сертификационных исследований», Москва

Получено 20.IV.2012

Рассматривается задача о числе различных $r$-грамм на циклах и отрезках линейных рекуррентных последовательностей над кольцами Галуа. Наибольший интерес результаты работы представляют для линейных рекуррент над примарными кольцами вычетов.

Ключевые слова: кольца Галуа, линейные рекуррентные последовательности, распределение элементов в последовательностях

The number of different $r$-patterns in linear recurrent sequences over Galois rings

\section{O. V. Kamlovskii}

LLC "Sertification Research Center", Moscow

Abstract. The number of different $r$-patterns on the period and on the path of period of linear recurrent sequences over Galois rings is discussed. These results are of interest for the linear recurrent sequences over residue rings of primary order.

Key words: Galois rings, linear recurrent sequences, distribution of elements of sequences

Citation: Mathematical Aspects of Cryptography, 2013, vol. 4, no. 3, pp. 49-82 (Russian). 


\section{Введение}

Важной и сложной задачей криптографии является изучение законов распределения элементов на отрезках псевдослучайных последовательностей. Часто при построении таких последовательностей используются линейные рекуррентные последовательности (ЛРП) над конечными полями и примарными кольцами вычетов (см., например, $[1,2])$. Вопрос о распределении элементов в ЛРП изучался многими авторами, однако в этом направлении существует большое количество нерешенных проблем. Сформулируем несколько таких задач. Пусть $u=(u(i))_{i=0}^{\infty}-$ ЛРП над коммутативным кольцом $R$ с единицей, $l$ - натуральное число, $M_{l}(u)$ - множество всех элементов кольца $R$ среди элементов $u(0), u(1), \ldots, u(l-1)$. Множество элементов кольца $R$ среди всех элементов ЛРП $u$ обозначим $M(u)$. Требуется: 1$)$ оценить величины $\left.\left|M_{l}(u)\right|,|M(u)|, 2\right)$ найти условия, при которых имеют место равенства $\left.M_{l}(u)=R, M(u)=R, 3\right)$ указать оценки значений $l$, при которых $M_{l}(u)=R$ или $M_{l}(u)=M(u)$.

Автору известна лишь одна работа [3], посвященная решению именно этих вопросов для ЛРП над конечными полями. Другие результаты в этом направлении были получены в [4-10] как следствия из решений других задач. Основной целью данной работы является решение указанных выше проблем для ЛРП над кольцами Галуа.

Сформулируем задачи в более общем виде. Пусть $u_{1}, \ldots, u_{r}-$ ЛРП над кольцом $R$ с одним и тем же характеристическим многочленом $F(x) \in R[x]$. Обозначим через $M_{l}\left(u_{1}, \ldots, u_{r}\right)\left(M\left(u_{1}, \ldots, u_{r}\right)\right)$ множество всех мультиграмм $\bar{z} \in R^{r}$, которые появляются среди $r$-грамм $\left(u_{1}(i), \ldots, u_{r}(i)\right)$, где $i=0,1, \ldots, l-1(i \geq 0)$. Для множеств $M_{l}\left(u_{1}, \ldots, u_{r}\right)$ и $M\left(u_{1}, \ldots, u_{r}\right)$ формулируются задачи, аналогичные изложенным выше пунктам 1)-3). Особый интерес представляет случай, когда последовательности $u_{1}, \ldots$, $u_{r}$ являются сдвигами одной ЛРП $u$. В этой ситуации элементы множеств $R^{r} \backslash M_{l}\left(u_{1}, \ldots, u_{r}\right)$ и $R^{r} \backslash M\left(u_{1}, \ldots, u_{r}\right)$ естественным образом связаны с запретными словами в выходных последовательностях фильтрующих генераторов, построенных на основе ЛРП $u$.

В данной работе в качестве кольца $R$ выбрано кольцо Галуа $R=$ $=G R\left(q^{n}, p^{n}\right)$, состоящее из $q^{n}$ элементов и имеющее характеристику $p^{n}$, где $p-$ простое число и $q=p^{t}$ для некоторого $t \in \mathbb{N}$. При $n=1$ кольцо $R$ совпадает с полем $G F(q)$, а при $t=1-$ с кольцом вычетов $\mathbb{Z}_{p^{n}}$. Это позволяет, с одной стороны, обобщить часть результатов, доказанных для ЛРП над полями, на случай ЛРП над кольцами Галуа, с другой стороны, получить важные следствия для ЛРП над примарными кольцами вычетов. 
Укажем две задачи, которые связаны с тематикой данной работы и для которых на данный момент нет удовлетворительного решения. Обозначим через $N_{l}\left(\bar{z}, u_{1}, \ldots, u_{r}\right)$ число появлений вектора $\bar{z} \in R^{r}$ среди $r$-грамм $\left(u_{1}(i), \ldots, u_{r}(i)\right)$, где $i=0,1, \ldots, l-1$. Величины $N_{l}\left(\bar{z}, u_{1}, \ldots, u_{r}\right)$ изучались в ряде работ (см., например, обзоры в [13] и [14]).

Очевидно, что множество $M_{l}\left(u_{1}, \ldots, u_{r}\right)$ совпадает с множеством тех векторов $\bar{z}$, для которых $N_{l}\left(\bar{z}, u_{1}, \ldots, u_{r}\right)>0$. Таким образом, некоторые свойства исследуемых множеств $M_{l}\left(u_{1}, \ldots, u_{r}\right)$ можно получить, используя известные оценки снизу для частот $N_{l}\left(\bar{z}, u_{1}, \ldots, u_{r}\right)$. В [15] изучалось расстояние единственности фильтрующего генератора над кольцом $R=G R\left(q^{n}, p^{n}\right)$, осуществляющего усложнение семейства $L_{R}(F)$ ЛРП с заданным характеристическим многочленом $F(x)$ с использованием функции усложнения $\Psi: R^{r} \rightarrow G F(q)$. Показано, что величина $l$ является верхней оценкой расстояния единственности рассматриваемого фильтрующего генератора, если для каждой ЛРП $u \in L_{R}(F) \backslash p L_{R}(F)$ в множестве $M_{l}\left(u, x u, \ldots, x^{r-1} u\right)$ имеется специальная серия из $n+1$ некоторых $r$-грамм. Связь изучаемых в данной работе параметров с задачей получения нижних оценок частот $r$-грамм в ЛРП, а также с задачей определения расстояний единственности фильтрующих генераторов показывает как важность предлагаемых исследований, так и их объективную сложность.

Работа имеет следующую структуру. В 11 на основе оценок частот $r$-грамм приводятся условия, при которых $M_{l}\left(u_{1}, \ldots, u_{r}\right)=R^{r}$ и $M\left(u_{1}, \ldots, u_{r}\right)=R^{r}$, если выполнены некоторые ограничения сверху на параметр $r$. В $\S 2$ рассматривается задача получения оценок снизу для величины $\left|M\left(u_{1}, \ldots, u_{r}\right)\right|$ при произвольных значениях $r$. Параграф 3 посвящен уточнению результатов $\S 2$ для частного случая $r=1$. Показано, что для кольца $R=\mathbb{Z}_{p^{n}}$ удается гарантировать равенство $M(u)=R$ при более слабых условиях, чем те, которые получены в 1 . В $\S 4$ приводятся нижние оценки для величины $\left|M_{l}\left(u_{1}, \ldots, u_{r}\right)\right|$, а также оценки снизу величины $\left|M\left(u_{1}, \ldots, u_{r}\right)\right|$, использующие другие параметры по сравнению с оценками из $\S 2$. Параграф 5 посвящен исследованию таких значений $l$, при которых $M_{l}\left(u_{1}, \ldots, u_{r}\right)=M\left(u_{1}, \ldots, u_{r}\right)$. В $\S 6$ уточняются результаты из $\S 5$ в частных случаях: при произвольном $r$ и $R=G F(q)$, а также при $r=1$ и $R=\mathbb{Z}_{p^{n}}$.

\section{§ 1. Некоторые следствия из оценок частот $\boldsymbol{r}$-грамм}

Представляет интерес рассмотрение таких ЛРП $u$ над кольцом $R$, для которых $M(u)=R$. Ясно, что если $u$ - чисто периодическая последовательность, т. е. длина ее подхода равна 0 , то $|M(u)| \leq T(u)$, где $T(u)-$ период по- 
следовательности $u$. Поэтому равенство $M(u)=R$ не выполнено для всех чисто периодических последовательностей $u$, у которых $T(u)<|R|$. Возникает вопрос о том, на сколько $T(u)$ должен быть больше $|R|$, чтобы гарантировать равенство $M(u)=R$ ? Частично на этот вопрос отвечает следующий результат из [8], полученный с использованием оценок частот элементов в ЛРП.

Утверждение 1. Пусть $F(x)$ - унитарный многочлен над полем $G F(q), F(0) \neq 0, \operatorname{deg} F(x)=m, u-Л P П$ над полем $G F(q)$ с характеристическим многочленом $F(x)$, у которой период $T(u)$ удовлетворяет условию

$$
T(u) \geq(q-1) q^{\frac{m}{2}} .
$$

Тогда выполнено равенство $M(u)=G F(q)$.

Таким образом, в ЛРП, имеющих достаточно большой период, появятся все элементы поля. В частности, при $m \geq 2$ условиям утверждения 1 удовлетворяют все ЛРП $u$ максимального периода над полем $G F(q)$.

Утверждение 1 допускает обобщение на случай $r$-грамм, но при более сильном ограничении на многочлен $F(x)$, а именно, он должен быть неприводим над полем $G F(q)$. Приведем этот результат в еще более общем виде. Назовем унитарный многочлен $F(x)$ над кольцом $R=G R\left(q^{n}, p^{n}\right)$ реверсивным многочленом Галуа, если его образ $\bar{F}(x)$ при действии естественного эпиморфизма колец $R[x] \rightarrow \bar{R}[x]$ неприводим над полем $\bar{R}=R / p R=G F(q)$ и $\bar{F}(x) \neq x$. Систему ЛРП $u_{1}, \ldots, u_{r}$ из множества $L_{R}(F)$ назовем линейно независимой над кольцом $R$, если для всех $\left(c_{1}, \ldots, c_{r}\right) \in R^{r} \backslash\{(0, \ldots, 0)\}$ последовательность $c_{1} u_{1}+\ldots+c_{r} u_{r}$ ненулевая. Для каждой последовательности $u \in R^{\infty}$ через $\bar{u}$ обозначим последовательность $\bar{u} \in \bar{R}^{\infty}$, полученную из $u$ заменой всех ее элементов на образы при действии естественного эпиморфизма колец $R \rightarrow \bar{R}$. Несложно заметить, что система $u_{1}, \ldots, u_{r}$ линейно независима над кольцом $R$ тогда и только тогда, когда система $\bar{u}_{1}, \ldots, \bar{u}_{r}$ линейно независима над полем $\bar{R}$. Если $F(x)$ - реверсивный многочлен Галуа, то каждая ЛРП $u \in L_{R}(F)$ является чисто периодической последовательностью и $T(u)$ делит период $T(F)$ многочлена $F(x)$. При этом в случае $u \in L_{R}(F) \backslash p L_{R}(F)$, т. е. $\bar{u} \neq(\overline{0})$, справедливо равенство $T(u)=T(F)$ [14].

Всюду в дальнейшем будем рассматривать реверсивный многочлен Галуа $F(x)$ и линейно независимую систему ЛРП $u_{1}, \ldots, u_{r}$ из множества $L_{R}(F)$, тогда $T\left(u_{1}\right)=\ldots=T\left(u_{r}\right)=T(F)$. Пусть $l-$ произвольное натуральное число. Введем обозначения:

$$
\begin{aligned}
& M_{l}\left(u_{1}, \ldots, u_{r}\right)= \\
& =\left\{\bar{z} \in R^{r}:\left(u_{1}(i), \ldots, u_{r}(i)\right)=\bar{z} \text { для некоторого } i \in\{0, \ldots, l-1\}\right\},
\end{aligned}
$$




$$
M\left(u_{1}, \ldots, u_{r}\right)=\left\{\bar{z} \in R^{r}:\left(u_{1}(i), \ldots, u_{r}(i)\right)=\bar{z} \text { для некоторого } i \in \mathbb{N}_{0}\right\} .
$$

В рассматриваемом случае справедливо равенство $M\left(u_{1}, \ldots, u_{r}\right)=$ $=M_{T(F)}\left(u_{1}, \ldots, u_{r}\right)$. Следующий результат из [16] обобщает утверждение 1 на класс реверсивных неприводимых многочленов над полем $G F(q)$.

Утверждение 2. Пусть $F(x)$ - реверсивный многочлен Галуа степени $m$ над кольцом $R=G R\left(q^{n}, p^{n}\right), u_{1}, u_{2}, \ldots, u_{r}-$ линейно независимая система ЛРП над кольцом $R$ с характеристическим многочленом $F(x)$. Тогда при выполнении условия

$$
T(\bar{F}) \geq p^{n-1}\left(q^{n r}-1\right) q^{\frac{m}{2}}
$$

имеет место равенство $M\left(u_{1}, \ldots, u_{r}\right)=R^{r}$.

В частности, если $\bar{F}(x)-$ многочлен максимального периода $T(\bar{F})=$ $=q^{m}-1$ над полем $G F(q)$, то он удовлетворяет условию утверждения 2 при $m \geq 2 n r+2(n-1) \log _{q} p$. Последнее неравенство накладывает следующее ограничение на $r: r \leq m /(2 n)$.

Приведем еще один результат из [16].

Утверждение 3. Если в условиях утверждения 2 число $l$ удовлетворяет неравенствам

$$
T(F) \geq l>\left(q^{n r}-1\right) p^{n+\nu-1}\left(\frac{2}{\pi} \ln T(F)+\frac{7}{5}\right) q^{\frac{m}{2}}
$$

mo $M_{l}\left(u_{1}, \ldots, u_{r}\right)=R^{r}$

Значит, при фиксированных параметрах $q, r, n$ и $m \rightarrow \infty$ равенство $M_{l}\left(u_{1}, \ldots, u_{r}\right)=R^{r}$ имеет место при $l \geq l^{*}=O\left(q^{m / 2} \ln T(F)\right)$.

\section{§2. Использование мультипликаторов многочленов}

Рассмотрим множество $M\left(u_{1}, \ldots, u_{r}\right)$, определенное равенством (2). Согласно утверждениям 1 и 2 при достаточно большом периоде многочлена $\bar{F}(x)$ выполнено равенство $M\left(u_{1}, \ldots, u_{r}\right)=R^{r}$. Однако не для всех значений $r$ удается обеспечить требуемый период $T(\bar{F})$. Представляет интерес получение нижних оценок мощности множеств $M\left(u_{1}, \ldots, u_{r}\right)$.

Будем использовать структурные свойства ЛРП, основанные на понятии мультипликатора ЛРП и связанного с ним понятия мультипликатора многочлена, которые использовались при решении различных задач (см., 
например, [17-19]). По аналогии с работой [20] назовем мультипликатором многочлена $F(x) \in R[x]$ над кольцом $R$ множество

$$
\operatorname{Mult}(F)=\left\{a \in R^{*}: F(x) \mid\left(x^{j}-a\right) \text { при некотором } j \geq 0\right\} .
$$

Изучим свойства мультипликатора реверсивного многочлена Галуа $F(x)$ степени $m$ над кольцом $R=G R\left(q^{n}, p^{n}\right)$. Известно (см. $\left.[11,12]\right)$, что в расширении $S=G R\left(q^{m n}, p^{n}\right)$ кольца $R$ содержится корень $\alpha$ многочлена $F(x)$, причем $\alpha \in S^{*}$. Группа $S^{*}$ является прямым произведением своих подгрупп $\Gamma(S)=\left\{x \in S: x^{q^{m}-1}=e\right\}$ и $e+p S$, причем группа $\Gamma(S)$ является циклической подгруппой порядка $q^{m}-1$. Пусть $\alpha=\alpha_{0} \beta$, где $\alpha_{0} \in \Gamma(S)$, $\beta \in e+p S$. Рассмотрим период $T(F)$ многочлена $F(x)$. Справедливы равенства $T(F)=p^{\nu} T(\bar{F})=p^{\nu}\left(q^{m}-1\right) / d$, где $0 \leq \nu \leq n-1$, а $d-$ делитель числа $q^{m}-1$. Кроме того, мультипликативные порядки $\operatorname{ord} \alpha_{0}$ и $\operatorname{ord} \beta$ элементов $\alpha_{0}$ и $\beta$ соответственно удовлетворяют равенствам ord $\alpha_{0}=T(\bar{F})$, a ord $\beta=p^{\nu}$. В случае, когда $R=\mathbb{Z}_{4}$ или $p>2$, группа $e+p S$ является прямым произведением подгруппы $e+p R$ и некоторой своей подгруппы $G$ (см. [11, глава 16]). В этом случае $\beta=\alpha_{1} \alpha_{2}$, где $\alpha_{1} \in e+p R$, а $\alpha_{2} \in G$, причем ord $\alpha_{1}=p^{k_{1}}$, ord $\alpha_{2}=p^{k_{2}}$ и $\max \left\{k_{1}, k_{2}\right\}=\nu$.

Для произвольной последовательности $u$ над кольцом $R$ обозначим через $P(u)$ множество всех последовательностей вида $x^{j} u$, где $j \in \mathbb{N}_{0}$. Множество $P(u)$ будем называть множеством всех сдвигов последовательности $u$.

Утверждение 4. Пусть $F(x)$ - реверсивный многочлен Галуа степени $m$ над кольиом $R=G R\left(q^{n}, p^{n}\right), T(F)=p^{\nu} T(\bar{F}), t=(T(\bar{F}), q-1)$. Тогда

1) $\operatorname{Mult}(F)=\langle\alpha\rangle \cap R^{*}$, где $\langle\alpha\rangle-$ группа, порожденная элементом $\alpha$;

2) для каждой ненулевой последовательности $u \in L_{R}(F)$ и элемента $a \in \operatorname{Mult}(F)$ выполнено включение aи $\in P(u)$, причем если $а и=x^{j} u$ для некоторого числа $j \in \mathbb{N}_{0}$, то $T(\bar{F}) / t$ делит $j$;

3) $|\operatorname{Mult}(F)|=t p^{\mu}$, где число $\mu=\mu(F)$ однозначно задается многочленом $F(x) u 0 \leq \mu \leq \nu$;

4) если либо $R-$ поле, либо $R=\mathbb{Z}_{4}$, либо $p>2$, то

$$
\mu(F)=\left\{\begin{array}{cc}
0 & \text { nрu } k_{1} \neq \nu \\
k_{1}-k_{2} & \text { nрu } k_{1}=\nu
\end{array}\right.
$$

Доказательство. 1) Достаточно заметить, что многочлен $F(x)$ делит многочлен $x^{j}-a$ для некоторого целого неотрицательного числа $j$ тогда и только тогда, когда $\alpha^{j}=a$. 
2) Представим ЛРП $u$ с помощью функции «след»

$$
u(i)=\operatorname{Tr}_{R}^{S}\left(b \alpha^{i}\right), \quad i \geq 0,
$$

где $b$ - однозначно определенный ненулевой элемент кольца $S$ (см. [12, теорема 8]). Если $a \in \operatorname{Mult}(F)$, то $a=\alpha^{j}$ для некоторого числа $j \geq 0$, и для последовательности $v=a u$ справедливы равенства

$$
\vartheta(i)=a \operatorname{Tr}_{R}^{S}\left(b \alpha^{i}\right)=\operatorname{Tr}_{R}^{S}\left(a b \alpha^{i}\right)=\operatorname{Tr}_{R}^{S}\left(b \alpha^{i+j}\right)=u(i+j) .
$$

Значит, $v=x^{j} u$ и $v \in P(u)$. Из равенства $a u=x^{j} u$ и соотношения (4) следует, что элемент $\alpha^{j}-a$ принадлежит множеству $p S$. Обозначим через $\bar{\alpha}$ образ элемента $\alpha$ при действии естественного эпиморфизма из кольца $S$ в поле $S / p S=G F\left(q^{m}\right)$, тогда элемент $\bar{\alpha}^{j}$ будет принадлежать полю $G F(q)$. Это возможно тогда и только тогда, когда $\operatorname{ord} \bar{\alpha}=T(\bar{F})$ делит $j(q-1)$, т.е. когда $T(\bar{F}) / t$ делит $j$.

3) С использованием п. 1 и равенства $R^{*}=\Gamma(R) \dot{\times}(e+p R)$ получим

$$
|\operatorname{Mult}(F)|=\left|\left\{j \in\{0, \ldots, T(F)-1\}: \alpha_{0}^{j} \in \Gamma(R), \beta^{j} \in e+p R\right\}\right| .
$$

Элемент $\alpha_{0}^{j}$ принадлежит $\Gamma(R)$ тогда и только тогда, когда $\alpha_{0}^{j(q-1)}=e$. Это условие равносильно тому, что $T(\bar{F}) / t$ делит $j$. В силу того, что ord $\beta=p^{\nu}$, выполнено равенство $|\langle\beta\rangle \cap(e+p R)|=p^{\mu}$ для некоторого $\mu=\mu(F) \leq \nu$. Элемент $\beta^{j}$ принадлежит множеству $e+p R$ тогда и только тогда, когда $\beta^{i p^{\mu}}=e$, т. е. число $p^{\nu-\mu}$ должно делить $j$. В итоге с использованием равенства (5) получим $|\operatorname{Mult}(F)|=t p^{\mu}$.

4) В рассматриваемом случае $e+p S=(e+p R) \dot{\times} G$, а значит, $\beta^{j}=$ $=\alpha_{1}^{j} \alpha_{2}^{j} \in e+p R$ тогда и только тогда, когда $\alpha_{2}^{j}=e$. Таким образом, если ord $\alpha_{2}=p^{\nu}$, то

$$
\operatorname{Mult}(F)=\left\{\alpha^{\frac{p^{\nu} T(\bar{F})}{t} s}, s=0,1, \ldots, t-1\right\},
$$

а если ord $\alpha_{2}=p^{k_{2}} \neq p^{\nu}$, то

$$
\operatorname{Mult}(F)=\left\{\alpha^{\frac{p^{k_{2} T(\bar{F})}}{t} s}, s=0,1, \ldots, t p^{\nu-k_{2}}-1\right\} .
$$

Заметим, что экспонента группы $e+p R$ равна $p^{n-1}$. Тогда, если в условиях утверждения 4 элемент $\widetilde{\beta}$ выбрать из группы $e+p R$ так, что $\operatorname{ord} \widetilde{\beta}=p^{k}$, где $k \in\{0, \ldots, \nu\}$, то элемент $\widetilde{\alpha}=\alpha_{0} \widetilde{\beta}$ удовлетворяет равенству $\overline{\widetilde{\alpha}}=\bar{\alpha}$. Это означает, что элемент $\widetilde{\alpha}$ будет корнем некоторого многочлена Галуа 
$G(x)$ степени $m$ над кольцом $R$, причем $\bar{G}(x)=\bar{F}(x), T(G)=p^{k} T(\bar{F})$ и $|\operatorname{Mult}(G)|=p^{k}(T(\bar{F}), q-1)$. Таким образом, в условиях утверждения 4 при каждом фиксированном многочлене $\bar{F}(x) \in \bar{R}[x]$ и каждом числе $\nu$ в классе всех многочленов Галуа $G(x)$ степени $m$ над кольцом $R$ таких, что $\bar{G}(x)=\bar{F}(x)$, параметр $\mu=\mu(G)$ пробегает все возможные значения от 0 до $\nu$.

В дальнейшем нам понадобится следующее вспомогательное утверждение, доказательство которого можно найти в [11, теорема 16.9] и [21, лемма 2.1].

Лемма 1. Пусть $R=G R\left(q^{n}, p^{n}\right), n \geq 2, \delta \in e+p R$, ord $\delta=p^{n-l}$, где $l \in\{1, \ldots, n\}$. Тогда

1) если $p>2$ или $p=n=2$, то $\delta=e+p^{l} r$, для некоторого элемента $r \in R^{*}$

2) если $p=2$ и $п>2$, то возможны два варианта:

a) $\delta=e+2^{l} r, l \geq 2, \delta^{2}=e+2^{l+1} r_{1}$, либо б) $\delta=e+2 r, \delta^{2}=e+2^{l+1} r_{1}$,

где $r, r_{1}-$ элементы из $R^{*}$.

Для каждого элемента $z$ кольца $R=G R\left(q^{n}, p^{n}\right)$ обозначим через $\|z\|$ его норму, которая равна такому наибольшему числу $s \in\{0, \ldots, n\}$, что $z \in p^{s} R$. Опишем порядок множества $z \operatorname{Mult}(F)$, состоящего из всех элементов вида $z b$, где $b \in \operatorname{Mult}(F)$.

Утверждение 5. Пусть $F(x)$ - реверсивный многочлен Галуа над кольцом $R=G R\left(q^{n}, p^{n}\right),|\operatorname{Mult}(F)|=t p^{\mu}$, где $t=(T(\bar{F}), q-1), z \in R,\|z\|=k$, где $k \in\{0, \ldots, n-1\}$. Тогда

1) при $p>2$ или $p=n=2$

$$
|z \operatorname{Mult}(F)|= \begin{cases}p^{\mu-k} t, & \text { если } k<\mu \\ t, & \text { если } k \geq \mu\end{cases}
$$

2) при $p=2, n>2$ возможны два случая:
a) $|z \operatorname{Mult}(F)|= \begin{cases}2^{\mu-k} t, & \text { если } k<\mu, \\ t, & \text { если } k \geq \mu ;\end{cases}$
б) $|z \operatorname{Mult}(F)|= \begin{cases}t, & \text { если } \mu=0 \text { или } k=n-1, \\ 2 t, & \text { если } \mu \geq 1, n-1>k \geq \mu-1, \\ 2^{\mu-k} t, & \text { если } \mu \geq 1, k<\mu-1 .\end{cases}$ 
Доказательство. Рассмотрим образующий элемент $\beta_{0} \beta_{1}$ группы $\operatorname{Mult}(F)$, где $\beta_{0} \in \Gamma(R), \beta_{1} \in e+p R$. Выясним, при каких значениях $i, j \in\left\{0,1, \ldots, p^{\mu} t-1\right\}$, где $i<j$, имеет место равенство

$$
z\left(\beta_{0} \beta_{1}\right)^{i}=z\left(\beta_{0} \beta_{1}\right)^{j} .
$$

В силу того, что $z=p^{k} \widetilde{z}$ для некоторого элемента $\widetilde{z} \in R^{*}$, равенство (6) равносильно выполнению включения $\left(\beta_{0} \beta_{1}\right)^{j-i} \in e+p^{n-k} R$. Отсюда следует, что $\beta_{0}^{j-i}=e$, а значит, найдется число $s \in \mathbb{N}_{0}$, удовлетворяющее условию $j=i+t s$. Тогда $\left(\beta_{0} \beta_{1}\right)^{j-i}=\delta^{s}$, где $\delta=\beta_{1}^{t}$. В силу взаимной простоты чисел $p$ и $q-1$ будем иметь ord $\delta=\operatorname{ord} \beta_{1}=p^{\mu}$. В итоге числа $i, j$ удовлетворяют равенству (6) тогда и только тогда, когда $j=i+t s$ и $\delta^{s} \in e+p^{n-k} R$ для некоторого $s \in \mathbb{N}$. Найдем числа $s \in \mathbb{N}$, при которых $\delta^{s} \in e+p^{n-k} R$.

1) Если $p>2$ или $p=n=2$, то $\delta=e+p^{n-\mu} r$, где $r \in R^{*}$, согласно лемме 1 . При $k \geq \mu$ имеем $\delta^{s} \in e+p^{n-k} R$ для всех $s \in \mathbb{N}$. Пусть $k<\mu$. Покажем, что $\delta^{s} \in e+p^{n-k} R$ тогда и только тогда, когда $p^{\mu-k}$ делит $s$. Представим $s$ в виде $s=p^{d} s_{1}$, где $d \in \mathbb{N}_{0},\left(s_{1}, d\right)=1$. Тогда $\delta^{s}=\left(\delta^{\prime}\right)^{p^{d}}$, где $\delta^{\prime}=\delta^{s_{1}}$. С использованием формулы бинома Ньютона получим $\delta^{\prime}=\left(e+p^{n-\mu} r\right)^{s_{1}}=e+p^{n-\mu} r^{\prime}$, где $r^{\prime} \in R^{*}$ и $\left(\delta^{\prime}\right)^{p^{d}}=\left(e+p^{n-\mu} r^{\prime}\right)^{p^{d}} \in e+p^{n-k} R$ тогда и только тогда, когда $d \geq \mu-k$. Таким образом, при $p>2$ утверждение доказано.

2) Пусть $p=2$ и $n>2$. Если в условии леммы 1 для элемента $\delta$ имеет место случай а), то доказательство повторяет проведенное в п. 1). Пусть в условии леммы 1 для элемента $\delta$ имеет место случай б). Если $k=n-1$ или $\mu=0$, то искомые числа $s$ любые. Рассмотрим случай, когда $k \leq n-2$ и $\mu \geq 1$. Если $\mu-1 \leq k \leq n-2$, то $\delta=e+2 r \notin e+2^{n-k} R$, а $\delta^{2}=e+2^{n-\mu+1} r_{1} \in$ $\in e+2^{n-k} R$. В этом случае искомые $s-$ это любые четные числа. Пусть $0 \leq k<\mu-1$. Если $s-$ нечетное число, то $\delta^{s}=(e+2 r)^{s}=e+2 r^{\prime}$, где $r^{\prime} \in R^{*}$ и $\delta^{s} \notin e+2^{n-k} R$. Рассмотрим $s=2^{d} s_{1}$, где $d, s_{1} \in \mathbb{N},\left(s_{1}, 2\right)=1$. С использованием формулы бинома Ньютона получим $\left(e+2^{n-\mu+1} r_{1}\right)^{s_{1}}=e+2^{n-\mu+1} r_{1}^{\prime}$, где $r_{1}^{\prime} \in R^{*}$ и $\delta^{s}=\left(e+2^{n-\mu+1} r_{1}\right)^{2^{d-1} s_{1}}=\left(e+2^{n-\mu+1} r_{1}^{\prime}\right)^{2^{d-1}} \in e+2^{n-k} R$ тогда и только тогда, когда $d \geq \mu-k$.

Пусть $F(x)$ - реверсивный многочлен Галуа над кольцом $R=$ $=G R\left(q^{n}, p^{n}\right)$. Зададим на множестве $R^{r}$ бинарное отношение $\sim$, положив для любых векторов $\bar{z}_{1}, \bar{z}_{2} \in R^{r} \bar{z}_{1} \sim \bar{z}_{2}$ тогда и только тогда, когда существует элемент $a \in \operatorname{Mult}(F)$ такой, что $\bar{z}_{1}=a \bar{z}_{2}$. Введенное отношение является отношением эквивалентности на множестве $R^{r}$. В дальнейшем для класса эквивалентности с представителем $\bar{z}$ будем использовать обозначение $[\bar{z}]_{\sim}$. Определим норму $\|\bar{z}\|$ вектора $\bar{z}=\left(z_{1}, z_{2}, \ldots, z_{r}\right)$ по правилу

$$
\|\bar{z}\|=\min \left\{\left\|z_{1}\right\|, \ldots,\left\|z_{k}\right\|\right\} .
$$

2013, T. 4, № 3, C. 49-82 
Тогда мощность класса $[\bar{z}]_{\sim}$ вычисляется с использованием утверждения 5 в силу справедливости равенства $\left|[\bar{z}]_{\sim}\right|=\left|z_{s} \operatorname{Mult}(F)\right|$, где $s$ выбрано так, что $\|\bar{z}\|=\left\|z_{s}\right\|$.

Теорема 1. Пусть $F(x)$ - реверсивный многочлен Галуа над кольияом $R=G R\left(q^{n}, p^{n}\right), u_{1}, u_{2}, \ldots, u_{r}$ - линейно независимая система ЛРП из множества $L_{R}(F),|\operatorname{Mult}(F)|=t p^{\mu}$, где $t=(T(\bar{F}), q-1)$. Тогда

1) для всех $\bar{z} \in M\left(u_{1}, \ldots, u_{r}\right)$ выполнено включение $[\bar{z}]_{\sim} \subset$ $\subset M\left(u_{1}, \ldots, u_{r}\right)$

2) $\left|M\left(u_{1}, \ldots, u_{r}\right)\right| \geq r p^{\mu} t$

3) если в множестве $M\left(u_{1}, \ldots, u_{r}\right)$ появляются векторы всех норм $k$, где $k=1,2, \ldots, n-1$, mo

$$
\left|M\left(u_{1}, \ldots, u_{r}\right)\right| \geq r p^{\mu} t+t\left(n-1-\mu+\frac{p^{\mu}-1}{p-1}\right) .
$$

Доказательство. 1) Пусть $\bar{z} \in M\left(u_{1}, \ldots, u_{r}\right)$ и число $i_{0} \in \mathbb{N}_{0}$ выбрано так, что $u_{s}\left(i_{0}\right)=z_{s}$ для всех $s=1,2, \ldots, r$. Тогда, как показано при доказательстве пункта 2 утверждения 4, для образующего элемента $a$ группы $\operatorname{Mult}(F)$ найдется такое $j \in \mathbb{N}_{0}$, что $u_{s}\left(i_{0}+j\right)=a u_{s}\left(i_{0}\right)$ для всех $s=1,2, \ldots, r$. Отсюда следует, что при всех $l \in \mathbb{N}_{0}$ имеет место равенство $\left(u_{1}\left(i_{0}+l j\right), \ldots, u_{r}\left(i_{0}+l j\right)\right)=a^{l} \bar{z}$, а значит, $[\bar{z}]_{\sim} \subset M\left(u_{1}, \ldots, u_{r}\right)$.

2) В силу линейной независимости системы $u_{1}, \ldots, u_{r}$ система их начальных векторов $\left(u_{1}(0), \ldots, u_{1}(m-1)\right), \ldots,\left(u_{r}(0), \ldots, u_{r}(m-1)\right)$ линейно независима над кольцом $R$. Рассмотрим матрицу

$$
U=\left(\begin{array}{cccc}
u_{1}(0) & u_{1}(1) & \ldots & u_{1}(m-1) \\
u_{2}(0) & u_{2}(1) & \ldots & u_{2}(m-1) \\
& & \ldots & \\
u_{r}(0) & u_{r}(1) & \ldots & u_{r}(m-1)
\end{array}\right)
$$

Матрица $\bar{U}$, полученная заменой всех элементов матрицы $U$ на их образы при действии естественного эпиморфизма из кольца $R$ в поле $\bar{R}=R / p R$, будет иметь ранг $r$. Отсюда следует, что матрица $U$ содержит $r$ столбцов, образующих линейно независимую систему над кольцом $R$. Значит, множество $M\left(u_{1}, \ldots, u_{r}\right)$ содержит систему $\bar{z}_{1}, \ldots, \bar{z}_{r}$ из $r$ линейно независимых векторов. В частности, все эти векторы имеют норму 0. По доказанному в п. 1 справедливо включение $\left[\bar{z}_{1}\right]_{\sim} \cup \ldots \cup\left[\bar{z}_{r}\right]_{\sim} \subset M\left(u_{1}, \ldots, u_{r}\right)$. Таким образом, $\left|M\left(u_{1}, \ldots, u_{r}\right)\right| \geq r|\operatorname{Mult}(F)|$. 
3) Обозначим через $\bar{s}_{k}$ вектор из множества $M\left(u_{1}, \ldots, u_{r}\right)$, имеющий норму $k$, где $k=1,2, \ldots, n-1$. Все векторы из класса $\left[\bar{s}_{k}\right]_{\sim}$ имеют норму $k$. С использованием утверждения 5 получим $\left|\left[\bar{s}_{k}\right]_{\sim}\right|=p^{\mu-k} t$ для всех $k=$ $=1, \ldots, \mu-1$ и $\left|\left[\bar{s}_{k}\right]_{\sim}\right| \geq t$ для всех $k=\mu, \ldots, n-1$. Из п. 1 следует, что $\left[\bar{s}_{1}\right]_{\sim} \cup \ldots \cup\left[\bar{s}_{n-1}\right]_{\sim} \subset M\left(u_{1}, \ldots, u_{r}\right)$. Тогда, учитывая классы $\left[\bar{z}_{1}\right]_{\sim}, \ldots,\left[\bar{z}_{r}\right]_{\sim}$, состоящие из векторов нормы 0 , получаем

$$
\begin{aligned}
& \left|M\left(u_{1}, \ldots, u_{r}\right)\right| \geq \\
& \geq r p^{\mu} t+p^{\mu-1} t+\ldots+p t+(n-\mu) t=r p^{\mu} t+t\left(n-1-\mu+\frac{p^{\mu}-1}{p-1}\right) .
\end{aligned}
$$

Отметим, что оценка из п. 2 теоремы 1 применима лишь в случае, когда известен параметр $\mu=\mu(F)$, который может принимать любые значения из отрезка $\{0, \ldots, \nu\}$, где $\nu$ определяется равенством $T(F)=p^{\nu} T(\bar{F})$. Если параметр $\mu$ не известен, то справедливо следствие из пункта 2 ): $\left|M\left(u_{1}, \ldots, u_{r}\right)\right| \geq r t$.

В оценке из п. 3 используется условие появления в множестве $M\left(u_{1}, \ldots, u_{r}\right)$ векторов всех норм, его проверка является отдельной задачей, решение которой не известно автору. Однако в некоторых случаях рассматриваемое условие выполняется. Например, при $m \geq n-1+r$ начальные векторы ЛРП $u_{1}, u_{2}, \ldots, u_{r}$ легко выбрать так, чтобы столбцы матрицы $U$, участвующей в доказательстве теоремы 1 , содержали векторы всех норм. В этом случае для оценки мощности множества $M\left(u_{1}, \ldots, u_{r}\right)$ будет применим п. 3 теоремы 1 .

В заключение этого параграфа приведем примеры, показывающие достижимость оценок из теоремы 1 . Пусть $d-$ простое число. Рассмотрим многочлен $F(x)=x^{d}-a$ над кольцом $R=G R\left(q^{n}, p^{n}\right)$. Согласно [13, теорема 3.75] многочлен $\bar{F}(x)=x^{d}-\bar{a}$ неприводим над полем $\bar{R}=R / p R=G F(q)$ тогда и только тогда, когда $\bar{F}(x)$ не имеет корней в поле $\bar{R}$. Пусть $d$ является делителем $q-1$ и элемент $a$ выбран так, чтобы $\bar{a}$ являлся примитивным элементом поля $\bar{R}$. В этом случае $F(x)$ является многочленом Галуа, причем $T(F)=d \operatorname{ord} a=d p^{\nu} \operatorname{ord} \bar{a}=p^{\nu} T(\bar{F})$, где $\nu \in\{0,1, \ldots, n-1\}$. Несложно проверить, что в этом случае $a$ является образующим группы $\operatorname{Mult}(F)$. Пусть $r \leq d-$ натуральное число. Рассмотрим ЛРП $u_{1}, u_{2}, \ldots, u_{r}$ из множества $L_{R}(F)$, имеющие следующие начальные векторы:

$$
(\underbrace{e, 0, \ldots, 0}_{r}, 0, \ldots, 0),(\underbrace{0, e, \ldots, 0}_{r}, 0, \ldots, 0), \ldots,(\underbrace{0,0, \ldots, e}_{r}, 0, \ldots, 0) \in R^{d} .
$$

Тогда $M\left(u_{1}, \ldots, u_{r}\right)=[(e, 0, \ldots, 0)]_{\sim} \cup[(0, e, \ldots, 0)]_{\sim} \cup \ldots \cup[(0,0, \ldots, e)]_{\sim}$, и оценка из п. 2 обращается в равенство. 
Выберем $d$, удовлетворяющее условию $d \geq r+n-1$, и рассмотрим такие ЛРП $u_{1}, \ldots, u_{r}$ из множества $L_{R}(F)$, что начальный вектор ЛРП $u_{1}$ равен

$$
(\underbrace{e, 0, \ldots, 0}_{r}, p e, p^{2} e, \ldots, p^{n-1} e, 0, \ldots, 0),
$$

а последовательности $u_{2}, \ldots, u_{r}$ совпадают с рассмотренными ранее. Тогда справедливо соотношение $M\left(u_{1}, \ldots, u_{r}\right)=[(e, \ldots, 0)]_{\sim} \cup \ldots \cup[(0, \ldots, e)]_{\sim} \cup$ $[(p e, \ldots, 0)]_{\sim} \cup \ldots \cup\left[\left(p^{n-1} e, \ldots, 0\right)\right]_{\sim}$. Согласно утверждению 5 это равенство показывает достижимость оценки из п. 3 для всех $p>2$.

\section{§3. Использование свойств разрядных последовательностей ЛРП}

В отличие от ЛРП максимального периода над конечными полями, для ЛРП максимального периода над примарными кольцами вычетов отсутствует полное описание частот появлений элементов. В настоящее время эта задача решена лишь для ЛРП над кольцом $\mathbb{Z}_{4}$ (см. [6] и [22]). Рассмотрим унитарный многочлен $F(x)$ максимального периода $T(F)=p^{n-1}\left(p^{m}-1\right)$ над кольцом $\mathbb{Z}_{p^{n}}$, где $\operatorname{deg} F(x)=m, n \geq 2$. Тогда найдутся многочлены $\Phi_{1}(x)$ и $\Phi_{2}(x)$ над кольцом $\mathbb{Z}_{p^{n}}$ такие, что

$$
x^{p^{m}-1} \equiv p \Phi_{1}(x) \quad(\bmod F)(x), \quad x^{p\left(p^{m}-1\right)} \equiv p^{2} \Phi_{2}(x) \quad(\bmod F)(x),
$$

причем многочлены $\bar{\Phi}_{1}(x)$ и $\bar{\Phi}_{2}(x)$, полученные из многочленов $\Phi_{1}(x)$ и $\Phi_{2}(x)$ приведением их коэффициентов по модулю $p$, будут ненулевыми многочленами над полем $\mathbb{Z}_{p}$, и будут справедливы соотношения $\operatorname{deg} \Phi_{1}(x)=m_{1}<m, \operatorname{deg} \Phi_{2}(x)=m_{2}<m$. Известен следующий результат:

Утверждение 6 ([6]). Пусть $F(x)$ - многочлен максимального периода над кольиом $R=\mathbb{Z}_{p^{n}}, u \in L_{R}(F), \bar{u} \neq(\overline{0})$. Тогда

1) если $p \geq 3, m_{1}=0$, то $R^{*} \subset M(u)$;

2) если $p \geq 3, m_{1} \neq 0$, то $M(u)=R$;

3) если $p=2, n \geq 3, m_{2}=0$, то $R^{*} \subset M(u)$;

4) если $p=2, n \geq 3, m_{2} \neq 0$, то $M(u)=R$.

В данном параграфе по аналогии с доказательством утверждения 6 будет рассмотрен более общий случай, когда $R=G R\left(q^{n}, p^{n}\right)$, а $F(x)-$ произвольный реверсивный многочлен Галуа над кольцом $R$. В частности, будет расширен класс ЛРП над кольцом $\mathbb{Z}_{p^{n}}$ для которых $M(u)=R$ или $R^{*} \subset M(u)$. 
Пусть $R=G R\left(q^{n}, p^{n}\right), F(x) \in R[x]-$ унитарный реверсивный многочлен, т. е. $F(x)$ - унитарный многочлен и $F(0) \in R^{*}$. Период $T(F)$ многочлена $F(x)$ связан с периодом многочлена $\bar{F}(x)$ соотношением $T(F)=p^{n-\lambda} T(\bar{F})$, где $\lambda \in\{1, \ldots, n\}$. Обозначим через $\stackrel{F}{=}$ отношение сравнимости по модулю многочлена $F(x)$. В дальнейшем нам понадобится следующая лемма, которая доказывается аналогично лемме 2 из [23, стр. 345].

Лемма 2. Пусть $F(x)$-реверсивный многочлен над кольияом $R=$ $=G R\left(q^{n}, p^{n}\right), T(F)=p^{n-\lambda} \tau_{0}$, где $\tau_{0}=T(\bar{F}), \lambda \in\{1, \ldots, n-1\}$. Тогда для всех $k \in\{1, \ldots, n-\lambda-1\}$ справедливы соотнотения

$$
x^{p^{k} \tau_{0}} \stackrel{F}{\equiv} e+p^{\lambda+k} \Phi_{k+1}(x),
$$

где $\Phi_{k+1}(x) \in R[x], \operatorname{deg} \Phi_{k+1}(x)<m, \bar{\Phi}_{2}(x)=\bar{\Phi}_{k+1}(x) \neq \overline{0}$. При этом если $p>2$ или $p=n=2$, то

$$
x^{\tau_{0}} \stackrel{F}{\equiv} e+p^{\lambda} \Phi_{1}(x)
$$

а если $p=2, n>2$, то возможен один из следующих вариантов:

либо а) $x^{\tau_{0}} \stackrel{F}{\equiv} e+2^{\lambda} \Phi_{1}(x), \lambda>1$, либо б) $x^{\tau_{0}} \stackrel{F}{\equiv} e+2 \Phi_{1}(x)$,

где $\Phi_{1}(x) \in R[x], \operatorname{deg} \Phi_{1}(x)<m, \Phi_{1}(x) \neq \overline{0}$. Кроме того, если $p>2$ или имеет место случай а), то $\bar{\Phi}_{1}(x)=\bar{\Phi}_{2}(x)$.

Разрядным множеством кольца $R=G R\left(q^{n}, p^{n}\right)$ называется любое его подмножество $K=\left\{k_{0}, k_{1}, \ldots, k_{q-1}\right\}$, все элементы которого попарно не сравнимы по идеалу $p R$ (см., например, [24]). Примером разрядного множества кольца $R$ является так называемое $p$-адическое множество $K=\Gamma(R)$, состоящее из всех таких элементов $r \in R$, что $r^{q}=r$. В кольце $\mathbb{Z}_{p^{n}}$ часто рассматривают так называемое $p$-ичное разрядное множество $K=\{0,1, \ldots, p-1\}$. Если $p=2$, то $p$-адическое и $p$-ичное разрядные множества совпадают. Число всех разрядных множеств кольца $R=G R\left(q^{n}, p^{n}\right)$ равно $|p R|^{q}=q^{q(n-1)}$.

Если $K-$ разрядное множество кольца $R=G R\left(q^{n}, p^{n}\right)$, то каждый элемент $a \in R$ однозначно представим в виде

$$
a=a_{0}+p a_{1}+p^{2} a_{2}+\ldots+p^{n-1} a_{n-1},
$$

где $a_{i} \in K, i=0,1, \ldots, n-1$. Равенство (7) называется разряднымм представлением элемента $a$ в разрядном множестве $K$, а элемент $a_{s}$, где $s=0,1, \ldots, n-1$, называется $s$-м разрядом элемента $a$. При этом элемент 
$a_{n-1}$ называется старшим разрядом. Для каждого $s \in\{1, \ldots, n\}$ рассмотрим отображение $\varkappa_{s}^{K}: R \rightarrow K$, определяемое для всех $a \in R$ по правилу

$$
\varkappa_{s}^{K}(a)=a_{s} .
$$

Зададим на множестве $K$ бинарные операции $\oplus$ и $\otimes$ :

$$
a \oplus b=\varkappa_{0}^{K}(a+b), \quad a \otimes b=\varkappa_{0}^{K}(a b)
$$

для всех $a, b \in K$. Тогда алгебра $(K, \oplus, \otimes)$ будет полем из $q$ элементов [24].

Пусть $u$ - последовательность над кольцом $R=G R\left(q^{n}, p^{n}\right), K-$ разрядное множество кольца $R$. Для каждого $s \in\{0, \ldots, n-1\}$ рассмотрим последовательность $u_{s}$ над полем $K$, полученную из последовательности $u$ по правилу $u_{s}(i)=\varkappa_{s}^{K}(u(i)), i \geq 0$. Последовательность $u_{s}$ будем называть $s$-й разрядной последовательностью последовательности $u$ в разрядном множестве $K$ и в этом случае будем использовать обозначение $u_{s}=\varkappa_{s}^{K}(u)$.

Укажем некоторые свойства разрядных последовательностей ЛРП $u$ над кольцом $R$. Будем использовать обозначение $\bar{u}$ для последовательности над полем $\bar{R}=R / p R$, полученной из последовательности $u$ заменой всех элементов на образы при действии естественного эпиморфизма колец $R \rightarrow \bar{R}$. Образ элемента $r \in R$ при действии этого эпиморфизма обозначим через $\bar{r}$. Если $u$ - последовательность над разрядным множеством $K$ кольца $R$, то, очевидно, имеет место равенство $T(u)=T(\bar{u})$. Кроме того, определим произведение многочлена $\bar{a}(x)=\sum_{i=0}^{l} \bar{a}_{i} x^{i} \in \bar{R}[x]$ и последовательности $u \in K^{\infty}$ по правилу $\bar{a}(x) u=\varkappa_{0}^{K}\left(a_{0} u\right) \oplus \varkappa_{0}^{K}\left(a_{0} x u\right) \oplus \ldots \oplus \varkappa_{0}^{K}\left(a_{l} x^{l} u\right)$. Несложно заметить, что многочлен $\bar{a}(x)$ аннулирует ЛРП $u \in K^{\infty}$ тогда и только тогда, когда этот многочлен аннулирует ЛРП $\bar{u}$.

Следующая теорема доказывается по аналогии с теоремой 1.3 из [24].

Теорема 2. Пусть $F(x)$ - реверсивный многочлен Галуа степени $m$ над кольиом $R=G R\left(q^{n}, p^{n}\right), T(F)=p^{n-\lambda} \tau_{0}$, где $\tau_{0}=T(\bar{F}), 1 \leq \lambda<n, u-$ ЛРП над кольцом $R$ с характеристическим многочленом $F(x), \bar{u} \neq(\overline{0}), K-$ разрядное множество кольиа $R, u_{s}=\varkappa_{s}^{K}(u), s=0,1, \ldots, n-1$. Тогда

1) если $p>2$, или $p=n=2$, или $p=2<n$, a $F(x)$ удовлетворяет условию а) леммы 2, то $T\left(u_{0}\right)=\tau_{0}, T\left(u_{r}\right) \mid \tau_{0}$ для всех $r \in\{1, \ldots, \lambda-1\}$,

$$
\left(x^{p^{k} \tau_{0}}-\bar{e}\right) \bar{u}_{\lambda+k}=\bar{\Phi}_{k+1}(x) \bar{u}=\bar{\Phi}_{1}(x) \bar{u}, \quad T\left(u_{\lambda+k}\right)=p^{k+1} \tau_{0},
$$

для всех $k \in\{0, \ldots, n-\lambda-1\}$; 
2) если $p=2<n u \quad F(x)$ удовлетворяет условию б) леммы 2, то $T\left(u_{0}\right)=\tau_{0}, T\left(u_{1}\right)=2 \tau_{0}, T\left(u_{r}\right) \mid 2 \tau_{0}$ для всех $r \in\{2, \ldots, \lambda\}$,

$\left(x^{\tau_{0}}-\bar{e}\right) \bar{u}_{1}=\bar{\Phi}_{1}(x) \bar{u},\left(x^{2^{k} \tau_{0}}-\bar{e}\right) \bar{u}_{\lambda+k}=\bar{\Phi}_{k+1}(x) \bar{u}=\bar{\Phi}_{2}(x) \bar{u}, T\left(u_{\lambda+k}\right)=2^{k+1} \tau_{0}$

для всех $k \in\{1, \ldots, n-\lambda-1\}$.

Отметим, что в теореме 2 не рассмотрен случай, когда $\lambda=n$, т.е. $T(F)=T(\bar{F})=\tau_{0}$. В этом случае ясно, что $T\left(u_{0}\right)=\tau_{0}$, а периоды $T\left(u_{1}\right), \ldots$, $T\left(u_{n-1}\right)$ делят $\tau_{0}$. Кроме того, заметим, что, как правило, при $\lambda<n$ имеет место равенство $T\left(u_{n-1}\right)=p^{n-\lambda} \tau_{0}=T(F)$. Исключение составляет случай, когда одновременно выполнены условия $p=2, n>2, \lambda=n-1$ и многочлен $F(x)$ удовлетворяет равенству из условия б) леммы 2 . В этом случае теорема 2 не позволяет установить равенство $T\left(u_{n-1}\right)=T(F)$.

Для последовательностей $a$ над полем $K$ и $b$ над полем $\bar{R}$ обозначим через $L(a, b)$ множество всех элементов $k \in K$, для которых найдутся такие число $i \in \mathbb{N}_{0}$ и элемент $\bar{r} \in \bar{R}, \bar{r} \neq \overline{0}$, что $(a(i), b(i))=(k, \bar{r})$.

Теорема 3. Пусть $F(x)$ - реверсивный многочлен Галуа степени $m$ над кольиом $R=G R\left(q^{n}, p^{n}\right), T(F)=p^{n-\lambda} \tau_{0}$, где $\tau_{0}=T(\bar{F}), 1 \leq \lambda<n$, $u \in L_{R}(F), \bar{u} \neq(\overline{0}), v=\Phi_{1}(x)$ u. Тогда если либо $p>2$, либо $p=n=2$, либо $p=2<n$, а $F(x)$ удовлетворяет условию а) леммы 2, то

$$
|M(u)| \geq\left|L\left(u_{0}, \bar{v}\right)\right| p^{n-\lambda} .
$$

Доказательство. Докажем методом математической индукции по параметру $l \in\{1,2, \ldots, n-\lambda\}$, что для каждого элемента $t_{0} \in L\left(u_{0}, \bar{v}\right)$ найдутся такая подгруппа $H$ порядка $p$ группы $(K, \oplus)$ и такие $l$ смежных классов по ней $k_{\lambda} \oplus H, \ldots, k_{\lambda+l-1} \oplus H$, что для любых $t_{\lambda} \in k_{\lambda} \oplus H, \ldots, t_{\lambda+l-1} \in k_{\lambda+l-1} \oplus H$ существует число $s \in \mathbb{N}_{0}$, удовлетворяющее соотношениям

$$
\bar{v}(s) \neq \overline{0}, H=\left\langle\varkappa_{0}^{K}(v(s))\right\rangle, u_{0}(s)=t_{0}, u_{\lambda}(s)=t_{\lambda}, \ldots, u_{\lambda+l-1}(s)=t_{\lambda+l-1} .
$$

Отсюда при $l=n-\lambda$, учитывая, что $|H|=p$, получим оценку $|M(u)| \geq$ $\geq\left|L\left(u_{0}, \bar{v}\right)\right| p^{n-\lambda}$.

Пусть $l=1, t_{0}-$ произвольный элемент из множества $L\left(u_{0}, \bar{v}\right)$ и пусть число $s^{\prime} \in \mathbb{N}_{0}$ удовлетворяет равенствам $u_{0}\left(s^{\prime}\right)=t_{0}, \bar{v}\left(s^{\prime}\right) \neq \overline{0}$. Так как $\left(x^{\tau_{0}}-\right.$ $-\bar{e}) \bar{u}_{\lambda}=\bar{v}$ согласно теореме 2 , то $\bar{u}_{\lambda}\left(i+\tau_{0}\right)=\bar{u}_{\lambda}(i)+\bar{v}(i)$ для всех $i \geq 0$. В частности, $\bar{u}_{\lambda}\left(s^{\prime}+j \tau_{0}\right)=\bar{u}_{\lambda}\left(s^{\prime}\right)+j \bar{v}(i)$ для всех $j=0,1, \ldots, p-1$. Отсюда и из изоморфизма полей $K$ и $\bar{R}$ следует, что

$$
\left\{u_{\lambda}\left(s^{\prime}+j \tau_{0}\right): j=0,1, \ldots, p-1\right\}=k_{\lambda} \oplus H,
$$


где $k_{\lambda}=u_{\lambda}\left(s^{\prime}\right)$, а $H-$ группа, порожденная элементом $\varkappa_{0}^{K}\left(v\left(s^{\prime}\right)\right)$. Тогда для любого элемента $t_{\lambda} \in k_{\lambda} \oplus H$ найдется такое число $j^{\prime} \in\{0,1, \ldots, p-1\}$, что $u_{\lambda}\left(s^{\prime}+j^{\prime} \tau_{0}\right)=t_{\lambda}$. В этом случае для элемента $s=s^{\prime}+j^{\prime} \tau_{0}$ с учетом равенств $T\left(u_{0}\right)=T(\bar{v})=\tau_{0}$ будем иметь $\bar{v}(s) \neq \overline{0}, H=\left\langle\varkappa_{0}^{K}(v(s))\right\rangle, u_{0}(s)=t_{0}$, $u_{\lambda}(s)=t_{\lambda}$.

Допустим, что утверждение верно для некоторого $l$; докажем, что оно верно при значении $l+1$, где $2<l+1 \leq n-\lambda$. По предположению индукции для каждого элемента $t_{0} \in L\left(u_{0}, \bar{v}\right)$ найдутся такая подгруппа $H$ порядка $p$ группы $(K, \oplus)$ и такие $l$ смежных классов по ней $k_{\lambda} \oplus H, \ldots, k_{\lambda+l-1} \oplus H$, что при любых $t_{\lambda} \in k_{\lambda} \oplus H, \ldots, t_{\lambda+l-1} \in k_{\lambda+l-1} \oplus H$ существует число $s^{\prime} \in \mathbb{N}_{0}$, удовлетворяющее соотношениям

$$
\begin{gathered}
\bar{v}\left(s^{\prime}\right) \neq \overline{0}, \quad H=\left\langle\varkappa_{0}^{K}\left(v\left(s^{\prime}\right)\right)\right\rangle, \\
u_{0}\left(s^{\prime}\right)=t_{0}, u_{\lambda}\left(s^{\prime}\right)=t_{\lambda}, \ldots, u_{\lambda+l-1}\left(s^{\prime}\right)=t_{\lambda+l-1} .
\end{gathered}
$$

Так как $\left(x^{p^{l} \tau_{0}}-\bar{e}\right) \bar{u}_{\lambda}=\bar{v}$ согласно теореме 2 , то $\bar{u}_{\lambda+l}\left(i+p^{l} \tau_{0}\right)=\bar{u}_{\lambda+l}(i)+$ $+\bar{v}(i)$ для всех $i \geq 0$. Тогда $\bar{u}_{\lambda+l}\left(s^{\prime}+j p^{l} \tau_{0}\right)=\bar{u}_{\lambda+l}(i)+j \bar{v}(i)$ для всех $j=$ $=0,1, \ldots, p-1$. Отсюда следует равенство

$$
\left\{u_{\lambda+l}\left(s^{\prime}+j p^{l} \tau_{0}\right): j=0,1, \ldots, p-1\right\}=k_{\lambda+l} \oplus H,
$$

где $k_{\lambda+l}=u_{\lambda+l}\left(s^{\prime}\right)$, а $H=\left\langle x_{0}^{K}\left(v\left(s^{\prime}\right)\right)\right\rangle$. Тогда для любого элемента $t_{\lambda+l} \in$ $\in k_{\lambda+l} \oplus H$ найдется такое число $j^{\prime} \in\{0,1, \ldots, p-1\}$, что $u_{\lambda+l}\left(s^{\prime}+j^{\prime} p^{l} \tau_{0}\right)=$ $=t_{\lambda+l}$. В этом случае для элемента $s=s^{\prime}+j^{\prime} p^{l} \tau_{0}$, учитывая, что каждое из чисел $T\left(u_{0}\right), T(\bar{v}), T\left(u_{\lambda}\right), \ldots, T\left(u_{\lambda+l-1}\right)$ делит $p^{l} \tau_{0}$, будем иметь $\bar{v}(s) \neq \overline{0}$, $H=\left\langle\varkappa_{0}^{K}(v(s))\right\rangle, u_{0}(s)=t_{0}, u_{\lambda}(s)=t_{\lambda}, \ldots, u_{\lambda+l}(s)=t_{\lambda+l}$.

Рассмотрим теперь множество $L\left(u_{0}, \bar{v}\right)$ из теоремы 3. Так как $\bar{v}-$ ненулевая последовательность, то данное множество непустое. Введем более удобное для изучения множество $L\left(\bar{u}_{0}, \bar{v}\right)$, состоящее из всех элементов $\bar{k} \in \bar{R}$, для которых найдется такой элемент $\bar{r} \in \bar{R}, \bar{r} \neq \overline{0}$, что пара $(\bar{k}, \bar{r})$ появляется среди биграмм $\left(\bar{u}_{0}(i), \bar{v}(i)\right)$. В силу изоморфизма полей $K$ и $\bar{R}$ справедливо равенство $\left|L\left(u_{0}, \bar{v}\right)\right|=\left|L\left(\bar{u}_{0}, \bar{v}\right)\right|$, причем $\bar{u}_{0}=\bar{u}$ и $\bar{v}$ являются ЛРП над полем $\bar{R}$ с характеристическим многочленом $\bar{F}(x)$.

Утверждение 7. В условиях теорем 2 и 3

1) если $\operatorname{deg} \bar{\Phi}_{1}(x)=0$, то $L(\bar{u}, \bar{v})=M(\bar{u}) \backslash\{\overline{0}\}$, и при условии $\tau_{0} \geq(q-$ - 1) $q^{\frac{m}{2}}$ справедливо равенство $L(\bar{u}, \bar{v})=\bar{R} \backslash\{\overline{0}\}$;

2) если $\operatorname{deg} \bar{\Phi}_{1}(x)>0$, то система $\bar{u}$, $\bar{v}$ линейно независима над полем $\bar{R}$, и при условии $\tau_{0} \geq\left(q^{2}-1\right) q^{\frac{m}{2}}$ имеет место равенство $L(\bar{u}, \bar{v})=\bar{R}$. 
Доказательство. 1) При справедливости равенства $\operatorname{deg} \bar{\Phi}_{1}(x)=0$ получим, что последовательности $\bar{u}$ и $\bar{v}=\bar{\Phi}_{1}(x) \bar{u}$ пропорциональны. Отсюда имеем $L(\bar{u}, \bar{v})=M(\bar{u}) \backslash\{\overline{0}\}$. Для завершения доказательства остается заметить, что согласно утверждению 1 неравенство $\tau_{0} \geq(q-1) q^{\frac{m}{2}}$ влечет равенство $M(\bar{u})=\bar{R}$.

2) При условии $\operatorname{deg} \bar{\Phi}_{1}(x)>0$ система последовательностей $\bar{u}, \bar{v}$ линейно независима над полем $\bar{R}$, и остается воспользоваться утверждением 2 .

Следствие 1. Пусть в условиях теорем 2 и $3 \lambda=1 u R=\mathbb{Z}_{p^{n}}$. Тогда 1) если $\operatorname{deg} \bar{\Phi}_{1}(x)=0 u \tau_{0} \geq(p-1) p^{\frac{m}{2}}$, то $R^{*} \subset M(u)$; 2) если $\operatorname{deg} \bar{\Phi}_{1}(x)>0$ $u \tau_{0} \geq\left(p^{2}-1\right) p^{\frac{m}{2}}$, mo $M(u)=R$.

Следствие 1 выводится непосредственно из теоремы 3 и утверждения 7.

Следствие 1 расширяет класс последовательностей (по сравнению с утверждением 6), для которых удается гарантировать появление всех обратимых элементов или всех элементов кольца в множестве $M(u)$. Кроме того, п. 2 следствия 1 при всех $n \geq 2$ накладывает более слабые условия на период многочлена $\bar{F}(x)$, чем утверждение 2 .

Применим оценки теоремы 3 к важному частному случаю, когда $\bar{F}(x)-$ многочлен максимального периода.

Следствие 2. Пусть выполнены в условия теорем 2 и $3, R=$ $=G R\left(q^{n}, p^{n}\right), \bar{F}(x)-$ многочлен степени $m$ над полем $\bar{R}$ периода $q^{m}-1$. Тогда

1) если $\operatorname{deg} \bar{\Phi}_{1}(x)=0$, то $|M(u)| \geq(q-1) p^{n-\lambda}$;

2) если $\operatorname{deg} \bar{\Phi}_{1}(x)>0, m \geq 2$, mо $|M(u)| \geq q p^{n-\lambda}$.

Доказательство. 1) Так как $\bar{F}(x)$ - многочлен максимального периода, то $M(\bar{u})=\bar{R}$, а значит, $L(\bar{u}, \bar{v})=\bar{R} \backslash\{\overline{0}\}$ по п. 1 утверждения 7.

2) По п. 2 утверждения 7 система последовательностей $\bar{u}, \bar{v}$ линейно независима над полем $\bar{R}$. Тогда при $m \geq 2$ среди биграмм $(\bar{u}(i), \bar{v}(i))$, где $i \geq 0$, появляются все биграммы из множества $\bar{R}^{2} \backslash\{(\overline{0}, \overline{0})\}$. Таким образом, $L(\bar{u}, \bar{v})=\bar{R}$.

В теореме 3 не рассмотрен случай, когда $p=2<n$, а многочлен $F(x)$ удовлетворяет условию б) леммы 2. Рассмотрим этот случай. Для последовательностей $a$ над полем $K$ и $b, c$ над полем $\bar{R}$ обозначим через $L(a, b, c)$ множество всех элементов $k \in K$, для которых существуют такие $i \in \mathbb{N}_{0}$ и элементы $\bar{r}_{1}, \bar{r}_{2} \in \bar{R} \backslash\{\overline{0}\}$, что $(a(i), b(i), c(i))=\left(k, \bar{r}_{1}, \bar{r}_{2}\right)$. 
Теорема 4. Пусть $n \in \mathbb{N}, n>2, F(x)$ - реверсивный многочлен Галуа степени $m$ над кольиом $R=G R\left(q^{n}, 2^{n}\right)$, удовлетворяющий условию б) леммы 2, $T(F)=2^{n-\lambda} \tau_{0}$, где $\tau_{0}=T(\bar{F}), 1 \leq \lambda<n, u \in L_{R}(F), \bar{u} \neq(\overline{0})$, $v=\Phi_{1}(x) u, \omega=\Phi_{2}(x) u$. Тогда

1) если $\lambda<n-1$, то

$$
|M(u)| \geq \max \left\{\left|L\left(u_{0}, \bar{\omega}\right)\right| 2^{n-\lambda-1},\left|L\left(u_{0}, \bar{v}, \bar{\omega}\right)\right| 2^{n-\lambda}\right\} ;
$$

2) если $\lambda=n-1$, то $|M(u)| \geq 2\left|L\left(u_{0}, \bar{v}\right)\right|$.

Доказательство. 1) Неравенство $|M(u)| \geq\left|L\left(u_{0}, \bar{\omega}\right)\right| 2^{n-\lambda-1}$ доказывается аналогично доказательству теоремы 3 с использованием соотношения $\left(x^{2^{l} \tau_{0}}-\bar{e}\right) \bar{u}_{\lambda+l}=\bar{\omega}$, где $l=1,2, \ldots, n-\lambda-1$. Доказательство неравенства $|M(u)| \geq\left|L\left(u_{0}, \bar{v}, \bar{\omega}\right)\right| 2^{n-\lambda}$ проводится аналогично доказательству теоремы 3 на основе равенств $\left(x^{\tau_{0}}-\bar{e}\right) \bar{u}_{1}=\bar{v}$ и $\left(x^{2^{l} \tau_{0}}-\bar{e}\right) \bar{u}_{\lambda+l}=\bar{\omega}$, где $l=1,2, \ldots, n-\lambda-1$.

2) Доказательство проводится аналогично доказательству теоремы 3 с использованием соотношения $\left(x^{\tau_{0}}-\bar{e}\right) \bar{u}_{1}=\bar{v}$.

Опишем некоторые свойства множества $L\left(u_{0}, \bar{v}, \bar{\omega}\right)$, участвующего в теореме 4. Для этого удобнее перейти к исследованию множества $L(\bar{u}, \bar{v}, \bar{\omega})$, состоящего из всех элементов $\bar{k} \in \bar{R}$, для которых найдутся такие элементы $\bar{r}_{1}, \bar{r}_{2} \in \bar{R} \backslash\{\overline{0}\}$, что тройка $\left(\bar{k}, \bar{r}_{1}, \bar{r}_{2}\right)$ появляется среди триграмм $(\bar{u}(i), \bar{v}(i), \bar{\omega}(i)), i \geq 0$. В силу изоморфизма полей $K$ и $\bar{R}$ выполнено равенство $\left|L\left(u_{0}, \bar{v}, \bar{\omega}\right)\right|=|L(\bar{u}, \bar{v}, \bar{\omega})|$, причем $\bar{u}_{0}=\bar{u}, \bar{v}, \bar{\omega}$ являются ЛРП над полем $\bar{R}$ с характеристическим многочленом $\bar{F}(x)$.

Утверждение 8. В условиях теорем 2 и 4

1) если выполнено хотя бы одно из условий

а) система $\bar{v}, \bar{\omega}$ линейно зависима,

б) система $\bar{v}$, $\bar{\omega}$ линейно независима и $\tau_{0} \geq\left(q^{2}-1\right) q^{\frac{m}{2}}$, то $L(\bar{u}, \bar{v}, \bar{\omega}) \neq \varnothing$

2) если система последовательностей $\bar{u}, \bar{v}, \bar{\omega}$ линейно независима $u \tau_{0} \geq\left(q^{3}-1\right) q^{\frac{m}{2}}$, mo $L(\bar{u}, \bar{v}, \bar{\omega})=\bar{R}$.

Доказательство. 1) При выполнении условия (а) ненулевые последовательности $\bar{v}, \bar{\omega}$ пропорциональны. Таким образом, $L(\bar{u}, \bar{v}, \bar{\omega}) \neq \varnothing$. Если выполнено условие (б), то $L(\bar{u}, \bar{v}, \bar{\omega}) \neq \varnothing$ согласно утверждению 2 .

2) При линейной независимости системы $\bar{u}, \bar{v}, \bar{\omega}$ с использованием утверждения 2 получим $L(\bar{u}, \bar{v}, \bar{\omega})=\bar{R}$. 
Следствие 3. Пусть выполнены условия теорем $2 u$ 4, $\lambda=1 u R=\mathbb{Z}_{2^{n}}$. Тогда

1) если $\operatorname{deg} \bar{\Phi}_{2}(x)=0 u \tau_{0} \geq 3 \cdot 2^{\frac{m}{2}}$, mо $R^{*} \subset M(u)$;

2) если $\operatorname{deg} \bar{\Phi}_{2}(x)>0 u \tau_{0} \geq 7 \cdot 2^{\frac{m}{2}}$, то $M(u)=R$.

Доказательство. Заметим, что при $\lambda=1$ и $R=\mathbb{Z}_{2^{n}}$ выполнены соотношения $\operatorname{deg} \bar{\Phi}_{1}(x)>0, \bar{\Phi}_{2}(x) \stackrel{\bar{F}}{\equiv} \bar{\Phi}_{1}(x)+\bar{\Phi}_{1}^{2}(x)$, где $\stackrel{\bar{F}}{\equiv}-$ отношение сравнимости по модулю многочлена $\bar{F}(x)$.

1) При выполнении условия $\bar{\Phi}_{2}(x)=\bar{e}$ справедливы соотношения $\bar{u}=\bar{\omega}, \bar{u} \neq \bar{v}$. С использованием пункта 1 утверждения 8 имеем $\bar{e} \in L(\bar{u}, \bar{v}, \bar{\omega})$, а значит, по теореме 4 множество $M(u)$ содержит $2^{n-1}$ различных обратимых элементов. Таким образом, $R^{*} \subset M(u)$.

2) В данном случае $\bar{v} \neq \bar{\omega}, \bar{u} \neq \bar{v}, \bar{u} \neq \bar{\omega}$. Равенство $\bar{u}=\bar{v}+\bar{\omega}$ не имеет места, так как приводит к противоречивому соотношению $\bar{F}(x) \mid\left(\bar{\Phi}_{1}^{2}(x)-\bar{e}\right)=$ $=\left(\bar{\Phi}_{1}(x)-\bar{e}\right)^{2}$. Значит, система $\bar{u}, \bar{v}, \bar{\omega}-$ линейно независима и по п. 2 утверждения 8 имеем $L(\bar{u}, \bar{v}, \bar{\omega})=\bar{R}$. В итоге с использованием теоремы 4 получим $M(u)=R$.

Следствие 3 описывает более широкий класс последовательностей (по сравнению с утверждением 6), для которых удается гарантировать соотношение $R^{*} \subset M(u)$ или равенство $M(u)=R$. Заметим также, что п. 2 следствия 3 при всех $n \geq 3$ накладывает более слабые условия на период многочлена $\bar{F}(x)$ по сравнению с утверждением 2.

Рассмотрим оценки из теоремы 4 применительно к важному частному случаю, когда $\bar{F}(x)-$ многочлен максимального периода.

Следствие 4. Пусть выполнены условия теорем 2 и 4, $R=G R\left(q^{n}, 2^{n}\right)$, $\bar{F}(x)-$ многочлен степени $m$ над полем $\bar{R}$ периода $q^{m}-1$, где $m \geq 2$. Тогда

1) при $\lambda<n-1$ :

a) если $\operatorname{deg} \bar{\Phi}_{1}(x)=0$ или $\operatorname{deg} \bar{\Phi}_{1}(x)>0, a \operatorname{deg} \bar{\Phi}_{2}(x)=0$, то $|M(u)| \geq(q-1) 2^{n-\lambda}$;

б) если $\operatorname{deg} \bar{\Phi}_{1}(x)>0 u \operatorname{deg} \bar{\Phi}_{2}(x)>0$, то $|M(u)| \geq q 2^{n-\lambda}$;

2) при $\lambda=n-1$ :

a) если $\operatorname{deg} \bar{\Phi}_{1}(x)=0$, то $|M(u)| \geq 2(q-1)$;

б) если $\operatorname{deg} \bar{\Phi}_{1}(x)>0$, то $|M(u)| \geq 2 q$.

Доказательство. 1) В случае а) последовательности $\bar{u}$ и $\bar{v}$ являются пропорциональными или последовательности $\bar{u}$ и $\bar{\omega}$ пропорциональны. Значит, $L(\bar{u}, \bar{v}, \bar{\omega})=\bar{R} \backslash\{\overline{0}\}$. В случае б) пары последовательностей $\bar{u}, \bar{v}$, а также $\bar{u}, \bar{\omega}$ не пропорциональны. Если последовательности $\bar{v}, \bar{\omega}$ пропорциональны, то, очевидно, $L(\bar{u}, \bar{v}, \bar{\omega})=\bar{R}$. Если $\bar{v}, \bar{\omega}$ не пропорциональны, то либо система 
$\bar{u}, \bar{v}, \bar{\omega}$ линейно независима, либо $\bar{u}=\overline{a v}+\bar{d} \bar{\omega}$ для некоторых ненулевых элементов $\bar{a}, \bar{d} \in \bar{R}$. В первом случае $m \geq 3$ и, очевидно, $L(\bar{u}, \bar{v}, \bar{\omega})=\bar{R}$, а во втором случае это равенство также выполнено, так как среди элементов вида $\bar{a} x_{1}+\bar{d} x_{2}$, где $x_{1}, x_{2} \in \bar{R} \backslash\{\overline{0}\}$, содержатся все элементы поля $\bar{R}$. Для завершения доказательства п. 1 остается воспользоваться теоремой 4. П. 2 непосредственно следует из теоремы 4.

Сравним оценки, полученные в этом параграфе, с оценками из 1 применительно к наиболее интересному случаю, когда $\bar{F}(x)$ - многочлен максимального периода $T(\bar{F})=q^{m}-1$ над полем $G F(q)$. В этом случае п. 2 теоремы 1 дает оценку

$$
|M(u)| \geq|\operatorname{Mult}(F)|=p^{\mu}(q-1) .
$$

Следствия 2 и 4 дают следующие оценки:

$$
|M(u)| \geq p^{n-\lambda}(q-1) \text { или }|M(u)| \geq p^{n-\lambda} q .
$$

Заметим, что параметр $\mu=\mu(F)$, как отмечалось ранее, не превосходит $n-\lambda$, поэтому оценки (9) не хуже оценки (8). Кроме того, как правило, имеет место вторая оценка в (9), существенно уточняющая неравенство (8). Однако оценка (8) применима при $\lambda=n$, чего нельзя сказать об оценках (9).

\section{§4. Нижние оценки числа $r$-грамм на отрезках}

Рассмотрим вопрос о числе $r$-грамм в множестве $M_{l}\left(u_{1}, \ldots, u_{r}\right)$, определенном равенством (1), при различных значениях длины $l$ рассматриваемого отрезка. В [3, теорема 3] был предложен один подход к получению нижних оценок величины $\left|M_{l}\left(u_{1}, \ldots, u_{r}\right)\right|$ для ЛРП над конечным полем. Обобщению этого результата на случай колец Галуа посвящен настоящий параграф.

Утверждение 9. Пусть и-ЛРП порядка т над коммутативным кольияом $R$, имеющая период $T(u)$ и подход $\Lambda(u)$. Тогда при всех таких $l$, что $m \leq l \leq T(u)+\Lambda(u)+m-1$, верно соотношение

$$
\left|M_{l}(u)\right| \geq(l-m+1)^{\frac{1}{m}}
$$

В частности, $|M(u)| \geq(T(u)+\Lambda(u))^{\frac{1}{m}}$.

Доказательство. Рассмотрим векторы $(u(i), u(i+1), \ldots, u(i+m-1))$, где $i \in\{0, \ldots, l-m\}$. Так как $l \leq T(u)+\Lambda(u)+m-1$, то эти векторы являются 
попарно различными элементами множества $M_{l}(u)^{m}$. Значит, $\left|M_{l}(u)\right| \geq(l-$ $-m+1)^{1 / m}$. Для получения второй оценки остается подставить в полученное неравенство значение $l=T(u)+\Lambda(u)+m-1$.

Обобщим утверждение 9 на случай произвольного $r$, добавив ограничения на многочлен $F(x)$.

Теорема 5. Пусть $F(x)$ - реверсивный многочлен Галуа степени $m$ над кольцом $R=G R\left(q^{n}, p^{n}\right), u_{1}, \ldots, u_{r}$-линейно независимая система ЛРП с характеристическим многочленом $F(x)$. Тогда при всех таких $l$, что $m-$ $-r+1 \leq l \leq T(F)+m-r$, верно неравенство

$$
\left|M_{l}\left(u_{1}, \ldots, u_{r}\right)\right| \geq(l-m+r)^{\frac{1}{m-r+1}} .
$$

В частности, $\left|M\left(u_{1}, \ldots, u_{r}\right)\right| \geq T(F)^{\frac{1}{m-r+1}}$.

Доказательство. Рассмотрим расширение $S=G R\left(q^{m n}, p^{n}\right)$ степени $m$ кольца $R$. В кольце $S$ многочлен $F(x)$ раскладывается на линейные множители $F(x)=\left(x-\alpha_{1}\right)\left(x-\alpha_{2}\right) \ldots\left(x-\alpha_{m}\right)$, причем образы $\bar{\alpha}_{1}, \ldots, \bar{\alpha}_{m}$ соответствующих корней многочлена $F(x)$ при действии естественного эпиморфизма $S \rightarrow S / p S=G F\left(q^{m}\right)$ являются попарно различными. Тогда согласно [25, предложение 5] для каждой ЛРП $u_{k}$, где $k=1,2, \ldots, r$, найдутся такие однозначно определенные элементы $a_{k 1}, a_{k 2}, \ldots, a_{k m}$ из кольца $S$, что

$$
u_{k}(i)=a_{k 1} \alpha_{1}^{i}+a_{k 2} \alpha_{2}^{i}+\ldots+a_{k m} \alpha_{m}^{i}, \quad i \geq 0 .
$$

Рассмотрим следующую систему линейных уравнений над кольцом $S$ :

$$
\left(\begin{array}{cccc}
a_{1 m} & a_{2 m} & \ldots & a_{r m} \\
a_{1 m-1} & a_{2 m-1} & \ldots & a_{r m-1} \\
& & \ldots & \\
a_{1 m-r+2} & a_{2 m-r+2} & \ldots & a_{r m-r+2}
\end{array}\right)\left(\begin{array}{c}
x_{1} \\
x_{2} \\
\vdots \\
x_{r}
\end{array}\right)=\left(\begin{array}{c}
0 \\
0 \\
\vdots \\
0
\end{array}\right) .
$$

Покажем, что эта система имеет решение

$$
c^{\downarrow}=\left(\begin{array}{c}
c_{1} \\
c_{2} \\
\vdots \\
c_{r}
\end{array}\right) \in S^{r}
$$

удовлетворяющее условию $\left\|c^{\downarrow}\right\|=\min \left\{\left\|c_{k}\right\|: k=1,2, \ldots, r\right\}=0$. Обозначим через $A$ основную матрицу этой системы. Согласно [26, утверждение 27 , 
стр. 258], матрица $A$ эквивалентна канонической матрице $K$ следующего вида:

$$
K=\operatorname{Diag}\left(p^{t_{1}} e, p^{t_{2}} e, \ldots, p^{t_{r-1}} e\right)_{r-1 \times r},
$$

где $t_{1}, t_{2}, \ldots, t_{r-1} \in \mathbb{N}_{0}$. Пусть $U, V$ - такие обратимые матрицы над кольцом $S$, что $K=U A V$. Тогда, если $y_{0}^{\downarrow}-$ решение системы $K y^{\downarrow}=0^{\downarrow}$, то $x_{0}^{\downarrow}=V y_{0}^{\downarrow}-$ решение системы $A x^{\downarrow}=0 \downarrow$. Если

$$
y_{0}^{\downarrow}=\left(\begin{array}{c}
0 \\
0 \\
\vdots \\
e
\end{array}\right),
$$

то вектор $c^{\downarrow}=V y_{0}^{\downarrow}$ является искомым решением исходной системы. Рассмотрим последовательность $\omega=c_{1} u_{1}+c_{2} u_{2}+\ldots+c_{r} u_{r}$. Для этой последовательности имеет место равенство

$$
\omega(i)=b_{1} \alpha_{1}^{i}+b_{2} \alpha_{2}^{i}+\ldots+b_{m-r+1} \alpha_{m-r+1}^{i}, \quad i \geq 0,
$$

где $b_{t}=c_{1} a_{1 t}+c_{2} a_{2 t}+\ldots+c_{r} a_{r t}$ для всех $t=1,2, \ldots, m-r+1$. Обозначим через $v_{t}$ последовательность над кольцом $S$, определенную равенством $v_{t}(i)=b_{t} \alpha_{t}^{i}, i \geq 0$. Тогда $\omega=v_{1}+v_{2}+\ldots+v_{m-r+1}$ и $\omega \in$ $\in L_{S}\left(\left(x-\alpha_{1}\right)\left(x-\alpha_{2}\right) \ldots\left(x-\alpha_{m-r+1}\right)\right)$. Отсюда следует, что $\omega$ является ЛРП порядка $m-r+1$. Покажем, что для ее периода $T(\omega)$ имеет место равенство $T(\omega)=T(F)$. Для каждого элемента $a \in S$ (последовательности $u$ над кольцом $S)$ обозначим через $\bar{a}(\bar{u})$ образ при действии естественного эпиморфизма $S \rightarrow \bar{S}=S / p S$. Из линейной независимости системы $u_{1}, \ldots, u_{r}$ над кольцом $R$ следует линейная независимость системы $\bar{u}_{1}, \ldots, \bar{u}_{r}$ над полем $\bar{S}$. В силу выбора вектора $\left(c_{1}, \ldots, c_{r}\right)$ последовательность $\bar{\omega}=\bar{c}_{1} \bar{u}_{1}+\ldots+\bar{c}_{r} \bar{u}_{r}-$ ненулевая ЛРП. Тогда из равенства

$$
\bar{\omega}(i)=\bar{b}_{1} \bar{\alpha}_{1}^{i}+\bar{b}_{2} \bar{\alpha}_{2}^{i}+\ldots+\bar{b}_{m-r+1} \bar{\alpha}_{m-r+1}^{i}, \quad i \geq 0,
$$

следует, что существует такое $j \in\{1,2, \ldots, m-r+1\}$, что $\bar{b}_{j} \neq \overline{0}$, т. е. $b_{j}-$ обратимый элемент кольца $S$. Так как последовательности $v_{1}, v_{2}, \ldots, v_{m-r+1}$ являются ЛРП с взаимно простыми характеристическими многочленами, то с использованием [23, утверждение 11, стр. 321] и равенств ord $\alpha_{1}=$ $=\ldots=\operatorname{ord} \alpha_{m-r+1}=T(F)$ получим $T(\omega)=\left[T\left(v_{1}\right), \ldots, T\left(v_{m-r+1}\right)\right]=$ $=T\left(v_{j}\right)=\operatorname{ord} \alpha_{j}=T(F)$. Доказательство теоремы завершает следующая цепочка неравенств: $\left|M_{l}\left(u_{1}, \ldots, u_{r}\right)\right| \geq\left|M_{l}(\omega)\right| \geq(l-m+r)^{\frac{1}{m-r+1}}$, справедливая по утверждению 9 для всех таких $l$, что $m-r+1 \leq l \leq T(F)+m-r$. 
Теорема 5 устанавливает единственную известную автору оценку снизу величины $\left|M_{l}\left(u_{1}, \ldots, u_{r}\right)\right|$ в случае колец Галуа. Отметим, что если рассмотреть $R=G F(q)$, то имеется другая оценка, установленная в [27]:

$$
\left|M_{l}\left(u_{1}, \ldots, u_{r}\right)\right| \geq \min \left\{\left|M\left(u_{1}, \ldots, u_{r}\right)\right|, l \cdot\left(\begin{array}{c}
p+m-r-1 \\
p-1
\end{array}\right)^{k}\right\},
$$

где $k=\log \left|M_{l}\left(u_{1}, \ldots, u_{r}\right)\right| / \log p$. Отсюда выводится оценка

$$
\left|M_{l}\left(u_{1}, \ldots, u_{r}\right)\right| \geq \min \left\{\left|M\left(u_{1}, \ldots, u_{r}\right)\right|, \frac{1}{p} \cdot l^{\frac{\log p}{\log p+p \log (m-r+1)}}\right\} .
$$

Вопрос о возможности обобщения оценки (10) на случай колец Галуа остается открытым.

Преимущество оценки величины $\left|M\left(u_{1}, \ldots, u_{r}\right)\right|$ из теоремы 5 по сравнению с оценками из теоремы 1 заключается в том, что она не требует знания мультипликатора многочлена $F(x)$, а требует лишь знания периода $T(F)$ многочлена $F(x)$. При $r=1$ и $T(F)=p^{\nu} T(\bar{F})=p^{\nu}\left(q^{m}-1\right)$ оценка из теоремы 5 точнее оценки из п. 2 теоремы 1 для всех $\nu \geq m \mu$. В частности, это так при $\mu=0$.

\section{Метод тригонометрических сумм}

В этом параграфе рассмотрим задачу нахождения значений $l$, при которых для величин, определенных равенствами (1) и (2), имеет место соотношение $M_{l}\left(u_{1}, \ldots, u_{r}\right)=M\left(u_{1}, \ldots, u_{r}\right)$.

Из неравенств (3) следует, что если $F(x)$ - реверсивный многочлен Галуа степени $m$ над кольцом $R=G R\left(q^{n}, p^{n}\right)$, то для каждой линейно независимой системы ЛРП $u_{1}, \ldots, u_{r}$ из множества $L_{R}(F)$ равенство $M_{l}\left(u_{1}, \ldots, u_{r}\right)=$ $=M\left(u_{1}, \ldots, u_{r}\right)=R^{r}$ имеет место при $l \geq l^{*}=O\left(q^{m / 2} \ln T(F)\right)$ и $m \rightarrow \infty$. В этом параграфе будет доказано более сильное утверждение, гарантирующее выполнение нужного равенства при $l^{*}=O\left(q^{m / 2}\right)$. Для этого по аналогии с результатами работ [9] и [28], относящимся к случаю $r=1$ и $R=G F(q)$, рассмотрим симметрическое уравнение специального вида.

Утверждение 10. Пусть $F(x)$ - реверсивный многочлен Галуа степени $m$ над кольияом $R=G R\left(q^{n}, p^{n}\right), T(F)=p^{\nu} T(\bar{F}), u_{1}, \ldots, u_{r}-$ линейно независимая система ЛРП с характеристическим многочленом $F(x), k \in \mathbb{N}$, 
$k \leq(T(F)+1) / 2, \bar{z}=\left(z_{1}, \ldots, z_{r}\right) \in R^{r}$. Тогда число $T_{k}\left(\bar{z}, u_{1}, \ldots, u_{r}\right)$ решений системы уравнений

$$
\left\{\begin{array}{c}
u_{1}(k-1+x-y)=z_{1}, \\
u_{2}(k-1+x-y)=z_{2}, \\
\cdots \\
u_{r}(k-1+x-y)=z_{r},
\end{array}\right.
$$

относительно неизвестных $x, y \in\{0, \ldots, k-1\}$ удовлетворяет условию

$$
\left|T_{k}\left(\bar{z}, u_{1}, \ldots, u_{r}\right)-\frac{k^{2}}{q^{n r}}\right| \leq \frac{q^{n r}-1}{q^{n r}} p^{n+\nu-1} k q^{\frac{m}{2}} .
$$

Доказательство. Каждому элементу $c$ кольца $R$ сопоставим характер $\chi_{c}$ группы $(R,+)$, определенный на элементах этой группы равенством

$$
\chi_{c}(r)=\exp \left\{2 \pi i \frac{\operatorname{Tr}_{R_{0}}^{R}(c r)}{p^{n}}\right\}
$$

где $R_{0}=\left\{0, e, 2 e, \ldots,\left(p^{n}-1\right) e\right\}-$ подкольцо кольца $R$, изоморфное кольцу $\mathbb{Z}_{p^{n}}, \operatorname{Tr}_{R_{0}}^{R}$ - функция «след» из кольца $R$ в кольцо $R_{0}$. Характер $\chi_{e}$ будем обозначать $\chi$. Справедливы следующие равенства (см., например, [16]):

$$
\sum_{c \in R} \chi_{c}(r)= \begin{cases}q^{n}, & \text { если } r=0 \\ 0, & \text { если } r \neq 0 .\end{cases}
$$

С использованием этих равенств получим

$$
T_{k}\left(\bar{z}, u_{1}, \ldots, u_{r}\right)=\sum_{x, y=0}^{k-1} \prod_{i=1}^{r} \frac{1}{q^{n}} \sum_{c_{i} \in R} \chi_{c_{i}}\left(u_{i}(k-1+x-y)-z_{i}\right) .
$$

Значит, справедливо равенство

$$
\begin{aligned}
& T_{k}\left(\bar{z}, u_{1}, \ldots, u_{r}\right)= \\
& =\frac{1}{q^{n r}} \sum_{x, y=0}^{k-1} \sum_{\left(c_{1}, c_{2}, \ldots, c_{r}\right) \in R^{r}} \chi\left(-\sum_{i=1}^{r} c_{i} z_{i}\right) \chi\left(\sum_{i=1}^{r} c_{i} u_{i}(k-1+x-y)\right) .
\end{aligned}
$$

Выделив в полученном равенстве отдельно слагаемое, соответствующее значению $\bar{c}=\left(c_{1}, c_{2}, \ldots, c_{r}\right)=\overline{0}$, и поменяв порядок суммирования, будем иметь

$$
T_{k}\left(\bar{z}, u_{1}, \ldots, u_{r}\right)=\frac{k^{2}}{q^{n r}}+\frac{1}{q^{n r}} \sum_{\bar{c} \in R^{r} \backslash\{\overline{0}\}} \chi(-\overline{c z}) \sum_{x, y=0}^{k-1} \chi\left(u_{\bar{c}}(k-1+x-y)\right),
$$


где для каждого вектора $\bar{c} \in R^{r}$

$$
\overline{c z}=\left(c_{1}, \ldots, c_{r}\right)\left(z_{1}, \ldots, z_{r}\right)=c_{1} z_{1}+\ldots+c_{r} z_{r},
$$

а $u_{\bar{c}}-$ последовательность элементов кольца $R$, определенная равенством

$$
u_{\bar{c}}(i)=c_{1} u_{1}(i)+\ldots+c_{r} u_{r}(i), \quad i \geq 0 .
$$

В силу линейной независимости системы $u_{1}, \ldots, u_{r}$ для всех $\bar{c} \neq \overline{0}$ последовательность $u_{\bar{c}}$ является ненулевой ЛРП с характеристическим многочленом $F(x)$. Тогда справедливо следующее неравенство:

$$
\left|T_{k}\left(\bar{z}, u_{1}, \ldots, u_{r}\right)-\frac{k^{2}}{q^{n r}}\right| \leq \frac{q^{n r}-1}{q^{n r}} \max _{u \in L_{R}(F), u \neq(0)}\left|\sum_{x, y=0}^{k-1} \chi(u(k-1+x-y))\right| .
$$

В дальнейшем будем оценивать модуль суммы

$$
\sigma_{k}(u)=\sum_{x, y=0}^{k-1} \chi(u(k-1+x-y)) .
$$

Пусть $T=T(F)$. Для каждого элемента $b \in \mathbb{Z}_{T}$ рассмотрим характер $\psi_{b}$ группы $\left(\mathbb{Z}_{T},+\right)$, задаваемый равенством $\psi_{b}(s)=\exp \left\{2 \pi i \frac{b s}{T}\right\}$ для всех $s \in \mathbb{Z}_{T}$. $\mathrm{C}$ использованием соотношения ортогональности для характеров получим

$$
\sum_{b=0}^{T-1} \psi_{b}(s)= \begin{cases}T, & \text { если } s=0 \\ 0, & \text { если } s \neq 0\end{cases}
$$

Тогда имеет место соотношение

$$
\sigma_{k}(u)=\frac{1}{T} \sum_{a=0}^{T-1} \chi(u(a)) \sum_{x, y=0}^{k-1} \sum_{b=0}^{T-1} \psi_{b}(a-(k-1+x-y)) .
$$

Изменив порядок суммирования, получим

$$
\sigma_{k}(u)=\frac{1}{T} \sum_{b=0}^{T-1} \psi_{b}(-(k-1)) \sum_{a=0}^{T-1} \chi(u(a)) \psi_{b}(a) \sum_{x, y=0}^{k-1} \psi_{b}(y-x) .
$$

Переходя к абсолютным величинам, получаем неравенства

$$
\left|\sigma_{k}(u)\right| \leq \frac{1}{T} \sum_{b=0}^{T-1}\left|\sum_{a=0}^{T-1} \chi(u(a)) \psi_{b}(a)\right|\left|\sum_{x, y=0}^{k-1} \psi_{b}(y-x)\right|
$$

2013, T. 4, № 3, C. 49-82 
Как показано в [16, утверждение 3], при всех $u \in L_{R}(F), u \neq(0)$, $b \in\{0, \ldots, T-1\}$ имеет место неравенство

$$
\left|\sum_{a=0}^{T-1} \chi(u(a)) \psi_{b}(a)\right| \leq p^{n+\nu-1} q^{\frac{m}{2}}
$$

Из него следует, что

$$
\left|\sigma_{k}(u)\right| \leq \frac{1}{T} p^{n+\nu-1} q^{\frac{m}{2}} \sum_{b=0}^{T-1}\left|\sum_{x, y=0}^{k-1} \psi_{b}(y-x)\right| .
$$

Кроме того, справедливы равенства

$$
\begin{gathered}
\sum_{b=0}^{T-1}\left|\sum_{x, y=0}^{k-1} \psi_{b}(y-x)\right|=\sum_{b=0}^{T-1}\left|\sum_{y=0}^{k-1} \psi_{b}(y) \sum_{x=0}^{k-1} \overline{\psi_{b}(x)}\right|=\sum_{b=0}^{T-1}\left|\sum_{g=0}^{k-1} \psi_{b}(g)\right|^{2}= \\
=\sum_{b=0}^{T-1} \sum_{x, y=0}^{k-1} \psi_{b}(y-x)=\sum_{x, y=0}^{k-1} \sum_{b=0}^{T-1} \psi_{b}(y-x)=k T .
\end{gathered}
$$

Таким образом, из неравенства (12) следует оценка $\left|\sigma_{k}(u)\right| \leq p^{n+\nu-1} k q^{\frac{m}{2}}$, и для завершения доказательства остается воспользоваться неравенством (11).

Теорема 6. Пусть $F(x)$ - реверсивный многочлен Галуа степени т над кольиом $R=G R\left(q^{n}, p^{n}\right), T(F)=p^{\nu} T(\bar{F}), u_{1}, \ldots, u_{r}-$ линейно независимая система ЛРП с характеристическим многочленом $F(x)$. Тогда если

$$
l>2\left(q^{n r}-1\right) p^{n+\nu-1} q^{\frac{m}{2}}
$$

$\operatorname{mo} M_{l}\left(u_{1}, \ldots, u_{r}\right)=M\left(u_{1}, \ldots, u_{r}\right)$.

Доказательство. Пусть число $l$ удовлетворяет условиям теоремы, $\bar{z}-$ произвольный элемент из множества $M\left(u_{1}, \ldots, u_{r}\right), i_{0}$ - наименьшее такое $i \in \mathbb{N}_{0}$, что

$$
\left\{\begin{array}{c}
u_{1}(i)=z_{1}, \\
u_{2}(i)=z_{2}, \\
\cdots \\
u_{r}(i)=z_{r} .
\end{array}\right.
$$


Если $i_{0}=0$, то $\bar{z} \in M_{l}\left(u_{1}, \ldots, u_{r}\right)$. Пусть $i_{0} \geq 1$ и $k=\left[\left(i_{0}+1\right) / 2\right]$. Тогда система уравнений

$$
\left\{\begin{array}{c}
u_{1}(k-1+x-y)=z_{1}, \\
u_{2}(k-1+x-y)=z_{2}, \\
\cdots \\
u_{r}(k-1+x-y)=z_{r}
\end{array}\right.
$$

не имеет решений при всех $x, y \in\{0, \ldots, k-1\}$, т. е. верно равенство $T_{k}\left(\bar{z}, u_{1}, \ldots, u_{r}\right)=0$. Тогда, согласно утверждению 10 , справедливы соотношения

$$
0=T_{k}\left(\bar{z}, u_{1}, \ldots, u_{r}\right) \geq \frac{k^{2}}{q^{n r}}-\frac{q^{n r}-1}{q^{n r}} p^{n+\nu-1} k q^{\frac{m}{2}} .
$$

Значит, $\left[\left(i_{0}+1\right) / 2\right]=k \leq\left(q^{n r}-1\right) p^{n+\nu-1} q^{m / 2}$. Таким образом, если $i_{0}$ нечетно, то $i_{0}+1 \leq 2\left(q^{n r}-1\right) p^{n+\nu-1} q^{\frac{m}{2}}<l$, а если $i_{0}$ четно, то $i_{0} \leq$ $2\left(q^{n r}-1\right) p^{n+\nu-1} q^{\frac{m}{2}}<l$. В итоге будет выполнено равенство $M_{l}\left(u_{1}, \ldots, u_{r}\right)=$ $M\left(u_{1}, \ldots, u_{r}\right)$.

\section{§5. Применение оценок рангов ЛРП}

Предложим теперь другой подход к оценке величин $l$, при которых все элементы, появляющиеся в множестве $M\left(u_{1}, \ldots, u_{r}\right)$, появятся среди элементов множества $M_{l}\left(u_{1}, \ldots, u_{r}\right)$. Данный подход является развитием идей, указанных в [7, теорема 4] и [10, лемма 11]. Пусть $R$ и $S$ - произвольные конечные коммутативные кольца с единицей и единица $e$ кольца $S$ отлична от нуля. Для каждой $r$-граммы $\bar{z} \in R^{r}$ рассмотрим отображение $\Phi_{\bar{z}}: R^{r} \rightarrow S$, задаваемое равенствами $\Phi_{\bar{z}}(\bar{z})=e, \Phi_{\bar{z}}(\bar{a})=0$ для всех $\bar{a} \in R^{r} \backslash\{\bar{z}\}$. Пусть $u_{1}, \ldots, u_{r}$ - произвольные ЛРП над кольцом $R$. Рассмотрим последовательность $v$ над кольцом $S$, элементы которой заданы равенством $v(i)=\Phi_{\bar{z}}\left(u_{1}(i), \ldots, u_{r}(i)\right), i \geq 0$. Всюду в дальнейшем для последовательности $v$ будем использовать обозначение $v=\Phi_{\bar{z}}\left(u_{1}, \ldots, u_{r}\right)$. В силу конечности кольца $R$ последовательности $u_{1}, \ldots, u_{r}$ являются периодическими. Отсюда следует, что последовательность $v$ также периодическая и, следовательно, она - ЛРП над кольцом $S$. Обозначим через rank v ранг последовательности $v$ (наименьшую из степеней всех ее характеристических многочленов над кольцом $S$ ).

Утверждение 11. При всех $l \in \mathbb{N}$, удовлетворяющии неравенству

$$
l \geq \max \left\{\operatorname{rank} \Phi_{\bar{z}}\left(u_{1}, \ldots, u_{r}\right): \bar{z} \in R^{r}\right\}
$$


справедливо соотношение $M_{l}\left(u_{1}, \ldots, u_{r}\right)=M\left(u_{1}, \ldots, u_{r}\right)$.

Доказательство. Пусть $\bar{z}-$ произвольная $r$-грамма из множества $M\left(u_{1}, \ldots, u_{r}\right)$, тогда последовательность $v=\Phi_{\bar{z}}\left(u_{1}, \ldots, u_{r}\right)$ является ненулевой ЛРП над кольцом $S$. Пусть $i_{0}-$ наименьшее целое неотрицательное число $i$, при котором $\left(u_{1}(i), \ldots, u_{r}(i)\right)=\bar{z}$. Тогда из определения последовательности $v$ следует равенство

$$
v=(\underbrace{0, \ldots, 0}_{i_{0}}, e, \ldots) .
$$

Отсюда следует, что $l \geq \operatorname{rank} v \geq i_{0}+1$, а значит, $\bar{z} \in M_{l}\left(u_{1}, \ldots, u_{r}\right)$.

Отметим, что в утверждении 11 не требуется, чтобы ЛРП $u_{1}, \ldots, u_{r}$ имели одинаковый характеристический многочлен и образовывали линейно независимую систему. Кроме того, не требуется, чтобы эти последовательности были чисто периодическими. Применим утверждение 11 к случаю $r=1$, $R=P=G F(q)$.

Теорема 7. Пусть $F(x)$ - унитарный многочлен степени $m$ над полем $P=G F(q), q=p^{t}$, где $p-$ простое число. Тогда для каждой ЛРП $и \in L_{P}(F)$

1) если $0 \in M(u)$ u $l>\left(\begin{array}{c}m+p-2 \\ p-1\end{array}\right)^{t}$, то $0 \in M_{l}(u)$,

2) при $l \geq\left(\begin{array}{c}m+p-1 \\ p-1\end{array}\right)^{t}-1$ выполнено равенство $M_{l}(u)=M(u)$.

Доказательство. Для каждого числа $j \in\{0, \ldots, q-1\}$ рассмотрим $p$-ичное представление $j=j_{0}+p j_{1}+\ldots+p^{t-1} j_{t-1}$, где $j_{0}, j_{1}, \ldots, j_{t-1} \in$ $\in\{0, \ldots, p-1\}$. Как показано в [29, теорема 4.1], для всех $j \in\{0, \ldots, q-1\}$ выполнено соотношение

$$
\operatorname{rank} u^{j} \leq \prod_{s=0}^{t-1}\left(\begin{array}{c}
m+j_{s}-1 \\
j_{s}
\end{array}\right) .
$$

1) Положим $S=P$ в утверждении 11 , тогда для отображения $\Phi_{0}$ справедливо равенство $\Phi_{0}(a)=e-a^{q-1}$. С использованием неравенства (13) получим

$$
\operatorname{rank} \Phi_{0}(u) \leq 1+\prod_{s=0}^{t-1}\left(\begin{array}{c}
m+p-2 \\
p-1
\end{array}\right)=1+\left(\begin{array}{c}
m+p-2 \\
p-1
\end{array}\right)^{t} .
$$

Тогда, как показано в доказательстве утверждения 11 , если $0 \in M(u)$, то

$$
l>\left(\begin{array}{c}
m+p-2 \\
p-1
\end{array}\right)^{t}
$$


2) Положим $S=P$ в утверждении 11 , тогда для каждого $z \neq 0$ отображение $\Phi_{z}$ задается равенством $\Phi_{z}(a)=e-(a-z)^{q-1}=b_{1} a+b_{2} a^{2}+$ $\ldots+b_{q-1} a^{q-1}$, где $b_{1}, b_{2}, \ldots, b_{q-1}-$ некоторые ненулевые элементы поля $P$. С помощью неравенства (13) получим

$$
\operatorname{rank} \Phi_{z}(u) \leq \sum_{j=1}^{q-1} \prod_{s=0}^{t-1}\left(\begin{array}{c}
m+j_{s}-1 \\
j_{s}
\end{array}\right) .
$$

В [30, следствие 1] показано, что

$$
\sum_{j=0}^{q-1} \prod_{s=0}^{t-1}\left(\begin{array}{c}
m+j_{s}-1 \\
j_{s}
\end{array}\right)=\left(\begin{array}{c}
m+p-1 \\
p-1
\end{array}\right)^{t} .
$$

Значит, $\operatorname{rank} \Phi_{z}(u) \leq\left(\begin{array}{c}m+p-1 \\ p-1\end{array}\right)^{t}-1$. В итоге с использованием п. 1 и утверждения 11 получим доказательство п. 2.

П. 2 теоремы 7 в отличие от теоремы 6 показывает, что длина $l$, при которой $M_{l}(u)=M(u)$, полиномиальна по параметру $m$, а именно, $l=O\left(m^{(p-1) \log _{p} q}\right)$ при фиксированном $q$ и $m \rightarrow \infty$.

Рассмотрим случай $r \geq 1, R=P=G F(q)$. Для чисел $m, s \in \mathbb{N}, s \geq 2$, $k \in \mathbb{N}_{0}$ обозначим через $\left\{\begin{array}{c}m \\ k\end{array}\right\}_{s}$ число решений уравнения $y_{1}+y_{2}+\ldots+y_{m}=k$ относительно целочисленных неизвестных $y_{i}$, удовлетворяющих условию $0 \leq y_{i} \leq s-1$ для всех $i \in\{1, \ldots, m\}$. Величина $\left\{\begin{array}{c}m \\ k\end{array}\right\}_{s}$ равна числу размещений $k$ одинаковых предметов по $m$ различным ячейкам при условии, что в каждую ячейку попадает не более $s-1$ предметов. Согласно [31, формула (3.22)]

$$
\left\{\begin{array}{l}
m \\
k
\end{array}\right\}_{s}=\sum_{i \geq 0}(-1)^{i}\left(\begin{array}{c}
m \\
i
\end{array}\right)\left(\begin{array}{c}
m+k-s i-1 \\
m-1
\end{array}\right) .
$$

Заметим, что справедливы соотношения

$$
\left(\begin{array}{l}
m \\
k
\end{array}\right)=\left\{\begin{array}{l}
m \\
k
\end{array}\right\}_{2} \leq\left\{\begin{array}{l}
m \\
k
\end{array}\right\}_{s} \leq\left\{\begin{array}{l}
m \\
k
\end{array}\right\}_{k+1}=\left(\begin{array}{c}
m+k-1 \\
k
\end{array}\right),
$$

кроме того, для всех $k=0,1, \ldots, s-1$

$$
\left\{\begin{array}{l}
m \\
k
\end{array}\right\}_{s}=\left(\begin{array}{c}
m+k-1 \\
k
\end{array}\right) \text {. }
$$

2013, T. 4, № 3, C. 49-82 
Теорема 8. Пусть $F(x)$ - реверсивный неприводимый многочлен степени $m$ над полем $P=G F(q), u_{1}, \ldots, u_{r}$ - ЛРП над полем $P$ с характеристическим многочленом $F(x), r \leq m$. Тогда при выполнении хотя бы одного из условий

$$
l \geq \sum_{k=0}^{r(q-1)}\left\{\begin{array}{l}
m \\
k
\end{array}\right\}_{q} \text { или } l \geq\left(\begin{array}{c}
m+r(q-1) \\
r(q-1)
\end{array}\right)
$$

будет выполнено равенство $M_{l}\left(u_{1}, \ldots, u_{r}\right)=M\left(u_{1}, \ldots, u_{r}\right)$.

Доказательство. Пусть выполнено первое условие. Согласно [1, утверждение 4, стр. 270], если степень многочлена, задающего отображение $\Phi: P^{r} \rightarrow P$, равна $d$, где $d \leq m(q-1)$, то

$$
\operatorname{rank} \Phi\left(u_{1}, \ldots, u_{r}\right) \leq \sum_{k=0}^{d}\left\{\begin{array}{l}
m \\
k
\end{array}\right\}_{q}
$$

Положим $S=P$ в утверждении 11 , тогда для всех $\bar{z}=\left(z_{1}, \ldots, z_{r}\right) \in P^{r}$ отображение $\Phi_{\bar{z}}$ задается многочленом $\left(e-\left(x_{1}-z_{1}\right)^{q-1}\right) \ldots\left(e-\left(x_{r}-z_{r}\right)^{q-1}\right)$, который имеет степень $r(q-1)$. Тогда с использованием неравенства (15) получим

$$
\operatorname{rank} \Phi_{\bar{z}}\left(u_{1}, \ldots, u_{r}\right) \leq \sum_{k=0}^{r(q-1)}\left\{\begin{array}{l}
m \\
k
\end{array}\right\}_{q},
$$

и остается воспользоваться утверждением 11.

В случае выполнения второго условия достаточно воспользоваться соотношениями

$$
\sum_{k=0}^{r(q-1)}\left\{\begin{array}{l}
m \\
k
\end{array}\right\}_{q} \leq \sum_{k=0}^{r(q-1)}\left(\begin{array}{c}
m+k-1 \\
k
\end{array}\right)=\left(\begin{array}{c}
m+r(q-1) \\
r(q-1)
\end{array}\right)
$$

Заметим, что второе условие теоремы 8 проще, но грубее первого условия этой теоремы. Однако при фиксированных параметрах $r, q$ и $m \rightarrow \infty$ обе нижние оценки эквивалентны и имеют вид $O\left(m^{r(q-1)}\right)$.

Пусть теперь $r=1, R=\mathbb{Z}_{p^{n}}$. В этом случае в условиях утверждения 11 удобнее всего выбрать $S=R / p R$ (см. [4]). Рассмотрим числа $b(k)$, где $0 \leq$ $\leq k \leq p^{n-1}$, определенные равенствами: $b(0)=p^{n}-1$, а для всех $k>0$

$b(k)=p^{n}-p k+\left\{\begin{array}{cl}\widetilde{k}-1, & \text { если } p \geq 3, \quad \text { где } \widetilde{1} \leqslant k \leqslant p-1: \widetilde{k} \equiv k(\bmod p-1), \\ 0, & \text { если } p=2, k \text { четно или } k=1, \\ 1, & \text { если } p=2, k \text { нечетно, } k \geq 3 .\end{array}\right.$ 
Теорема 9 ([4]). Пусть $F(x)$ - реверсивный многочлен Галуа степени $m$ над примарным кольцом вычетов $R=\mathbb{Z}_{p^{n}}, u \in L_{R}(F), \bar{u} \neq(\overline{0})$. Тогда при

$$
l \geq \sum_{i=0}^{p^{n-1}-1}(i+1) \sum_{k=b(i+1)+1}^{b(i)}\left\{\begin{array}{l}
m \\
k
\end{array}\right\}_{p}
$$

выполнено равенство $M_{l}(u)=M(u)$.

Отметим, что при $n=1$ теорема 9 устанавливает тот же самый результат, что и п. 2 теоремы 7 для случая $t=1$.

Остановимся на наиболее интересном случае $p=2$ и упростим оценку из теоремы 9.

Следствие 5. Пусть выполнены условия теоремы 9, $p=2, m \geq 3$, $n \geq 3$. Тогда при

$$
l \geq\left(\begin{array}{c}
m+2^{n}-2 \\
2^{n}-1
\end{array}\right)
$$

выполнено соотношение $M_{l}(u)=M(u)$.

Доказательство. Покажем, что при $m \geq 3$ и $n \geq 3$ правая часть неравенства (17) не меньше правой части неравенства (16). Непосредственной проверкой легко убедиться, что при $m=3$ правая часть неравенства (17) не меньше правой части неравенства (16). Пусть теперь $m \geq 4$. Правая часть неравенства (16) не превосходит величины

$$
\sum_{i=0}^{2^{n}-4}\left(\begin{array}{c}
m \\
2^{n}-1-i
\end{array}\right)(i+1)+2^{n-1}\left(\begin{array}{c}
m \\
2
\end{array}\right)+2^{n-1}\left(\begin{array}{c}
m \\
1
\end{array}\right) .
$$

Методом математической индукции по параметру $s \in\{0, \ldots, l\}$ несложно показать, что если $s, l, k, m$ целые неотрицательные числа и $s \leq l \leq k$, то

$$
\left(\begin{array}{c}
m+l \\
k
\end{array}\right)=\sum_{i=0}^{s}\left(\begin{array}{c}
m+l-s \\
k-i
\end{array}\right)\left(\begin{array}{l}
s \\
i
\end{array}\right)
$$

Подставляя в равенство (19) значения $l=s=2^{n}-2, k=2^{n}-1$, получаем

$$
\left(\begin{array}{c}
m+2^{n}-2 \\
2^{n}-1
\end{array}\right)=\sum_{i=0}^{2^{n}-2}\left(\begin{array}{c}
m \\
2^{n}-1-i
\end{array}\right)\left(\begin{array}{c}
2^{n}-2 \\
i
\end{array}\right) .
$$

2013, T. 4, № 3, C. 49-82 
С использованием равенства (19) получим, что при всех $i \in\left\{0, \ldots, 2^{n}-4\right\}$ справедливы соотношения

$$
\left(\begin{array}{c}
2^{n}-2 \\
i
\end{array}\right)=\sum_{j=0}^{i}\left(\begin{array}{c}
2^{n}-2-i \\
i-j
\end{array}\right)\left(\begin{array}{l}
i \\
j
\end{array}\right) \geq i+1 .
$$

Кроме того, при $m \geq 4$ справедливы неравенства

$$
\left(\begin{array}{c}
m \\
2
\end{array}\right)\left(\begin{array}{l}
2^{n}-2 \\
2^{n}-3
\end{array}\right)+\left(\begin{array}{c}
m \\
1
\end{array}\right)\left(\begin{array}{l}
2^{n}-2 \\
2^{n}-2
\end{array}\right) \geq 2^{n-1}\left(\left(\begin{array}{c}
m \\
2
\end{array}\right)+\left(\begin{array}{c}
m \\
1
\end{array}\right)\right) .
$$

Поэтому правая часть неравенства (16) не меньше величины (18), а значит, и правой части неравенства (16).

Оценка из следствия 5 проще, но грубее соответствующей оценки из теоремы 9. Однако при фиксированном $n$ и $m \rightarrow \infty$ обе оценки эквивалентны и имеют вид $O\left(m^{2^{n}-1}\right)$.

Результаты утверждения 11 не удается применить к случаю, когда рассматриваются $r$-граммы, где $r>1$, а кольцо $R$ не является полем. Если $R$ - коммутативное кольцо с единицей, то известны оценки рангов ЛРП $v=\Phi\left(u_{1}, \ldots, u_{r}\right)$ в случае, когда отображение $\Phi: R^{r} \rightarrow R$ задается многочленом (см. [25]). Однако рассматриваемые в утверждении 11 отображения $\Phi_{\bar{z}}$, как правило, не представимы многочленами над кольцом $R$. Несложно показать, что если $R=\mathbb{Z}_{p^{n}}$, где $n>1$, то отображение $\Phi_{\bar{z}}$ задается полиномом тогда и только тогда, когда $R=\mathbb{Z}_{4}$ и $r=1$.

\section{Список литературы}

1. Алферов А.П., Зубов А.Ю., Кузьмин А.С., Черемушкин А.В. Основы криптографии: Учебное пособие. - М.: Гелиос АРВ, 2001. - 480 с.

2. Кнут Д. Э. Искусство программирования: Учебное пособие. Т. 2. - М.: Изд. дом Вильямс, 2000. - 832 с.

3. Mullen G. L., Shparlinski I. E. Values of linear recurring sequences of vectors over finite fields // Acta Arith. - 1993. - V. 65. № 3. - P. 221-226.

4. Былков Д. Н., Камловский О.В. Индексы вхождений элементов в линейные рекуррентные последовательности над примарными кольцами вычетов // Проблемы передачи информации. - 2008. - Т. 44. № 2. C. $101-109$.

5. Камловский О.В., Кузьмин А.С. Оценки частот появления элементов в линейных рекуррентных последовательностях над кольцами Галуа // Фундам. и прикл. математика. - 2000. - Т. 6. № 4. - С. 1083-1094. 
6. Кузьмин, А. С. Распределение элементов на циклах линейных рекуррент над кольцами вычетов // Успехи матем. наук. - 1992. - Т. 47. № 6. С. 213-214.

7. Шиарлинский И. Е. О распределении значений рекуррентных последовательностей // Проблемы передачи информации. -1989 . - Т. 25. № 2 . C. $46-53$.

8. Chou W.S., Mullen G.L. Generating linear spans over finite fields // Acta Arith. - 1992. - V. 61. № 2. - P. 183-191.

9. Шпарлинский И.Е. О числе простых делителей рекуррентных последовательностей // Матем. заметки. - 1985. - Т. 38. № 1. C. 29-34.

10. Шnарлинский И. Е. О числе различных простых делителей рекуррентных последовательностей // Матем. заметки. - 1987. - Т. 42. № 4. - С. $494-$ 507.

11. McDonald B.R. Finite rings with identity. New York. Dekker. - 1974. $429 \mathrm{p}$.

12. Нечаев A. А. Код Кердока в циклической форме // Дискретн. матем. 1989. - Т. 1. № 4. - С. 123-139.

13. Лидл Р., Нидеррайтер Г. Конечные поля. Т. 1, 2. - М.: Мир, 1988. - 824 с.

14. Кузьмин А. С., Куракин В. Л., Нечаев А. А. Псевдослучайные и полилинейные последовательности // В сб.: Труды по дискретной математике, т. 1. М.: ТВП, 1997. - С. 139-202.

15. Былков Д.Н. Класс усложнений линейных рекуррент над кольцом Галуа, не приводящий к потере информации // Проблемы передачи информации. -2010 . - Т. 46. № 3. - С. 51-59.

16. Камловский $О . B$. Частотные характеристики линейных рекуррентных последовательностей над кольцами Галуа // Матем. сборник. - 2009. T. 200. № 4. - C. 31-52.

17. Hall M. Equidistribution of residues in sequences // Duke Math J. - 1938. V. 4. № 4. - P. 691-695.

18. Лаксов Д. Линейные рекуррентные последовательности над конечными полями // Математика. Сборник переводов. - 1967. - Т. 11. № 6. C. $145-158$.

19. Нечаев А.А., Кузьмин А.С., Куракин В.Л. Структурные, аналитические и статистические свойства линейных и полилинейных рекуррент // В сб.: Труды по дискретной математике, т. 3. - М.: ТВП, 2000. - С. 155-194.

20. Михайлов Д.А. Унитарные полилинейные регистры и их периоды // Дискретн. матем. - 2002. - Т. 14. Вып. 1. - С. 30-59. 
21. Нечаев А. А. Цикловые типы линейных подстановок над конечными коммутативными кольцами // Матем. сборник. - 1993. - Т. 184. № 3. C. 21-56.

22. Kuzmin A.S., Nechaev A.A. Complete weight enumerators of generalized Kerdock code and related linear codes over Galois ring // Discr. Appl. Math. 2001. - V. 111. - P. 117-137.

23. Глухов М. М., Елизаров В.П., Нечаев А.А. Алгебра. Т. 2. - М.: Гелиос APB, 2003. $-414 \mathrm{c}$.

24. Кузьмин А.С., Нечаев А. А. Линейные рекуррентные последовательности над кольцами Галуа // Алгебра и логика. - 1995. - Т. 34. № 2. - С. 169 189.

25. Куракин В.Л. Полиномиальные преобразования линейных рекуррентных последовательностей над конечными коммутативными кольцами // Дискретн. матем. - 2000. - Т. 12. Вып. 3. - С. 3-36.

26. Елизаров В. П. Конечные кольца. - М.: Гелиос АРВ, 2006. - 304 с.

27. Shparlinski I.E. On the distribution of values of recurring sequences and the Bell numbers in finite fields // European J. Combin. - 1991. - V. 12. P. 81-87.

28. Tietavainen $A$. On the solvability of equations in incomplete finite fields // Ann. Univ. Turkuensis. - 1967. - V. 102. - P. 3-12.

29. Herlestam T. On functions of linear shift register sequences // Lect. Notes Comput. Sci. - 1986. - V. 219. - P. 119-129.

30. Куракин В. Л. Первая координатная последовательность линейной рекурренты максимального периода над кольцом Галуа // Дискретн. матем. 1994. - Т. 6. № 2. - С. 88-100.

31. Сачков В.Н. Введение в комбинаторные методы дискретной математики. - М.: Наука, 1982. - 384 с. 